Check for updates

Cite this: Mater. Adv., 2022, 3,2568

Received 8th December 2021 Accepted 26th January 2022

DOI: $10.1039 / \mathrm{d} 1 \mathrm{ma01160j}$

rsc.li/materials-advances

\section{Smart DEA-QCGM-CNT hydrogels with temperature- and NIR-responsive behavior achieved by the synergy between CNT and QCGM for wound dressing}

\author{
Qing Zhong, ${ }^{a}$ Gen $\mathrm{Li}^{\mathrm{a}}{ }^{\mathrm{H}}$ Hui Long, ${ }^{\mathrm{a}}$ Jingxian Zhang, ${ }^{\text {a Suiping Deng, }}{ }^{\mathrm{a}}$ \\ Langhuan Huang (D) *a and Shaozao Tan (D) *ab
}

\begin{abstract}
Temperature-sensitive hydrogels are always hotspot and have shown great potential in the field of biomedicine. Herein, we report $N, N$-diethylacrylamide (DEA) temperature-sensitive nanocomposite hydrogels with improved mechanical properties composed of carbon nanotubes (CNT) and glycidyl methacrylate functionalized quaternized chitosan (QCGM), followed by deciphering the synergy between CNT and QCGM for the influence on the properties of the hydrogels. The prepared hydrogels not only present a temperature-stimulus response but also feature in NIR-stimulus response performance due to the addition of $\mathrm{CNT}$. In particular, the $\mathrm{DQ}_{0.5} \mathrm{C}_{1}$ hydrogel exhibits the optimal performance, including high mechanical strength and thermal behavior, and was subjected to examine the photothermal conversion ability, as well as loading the model drug ciprofloxacin hydrochloride (CIP) to investigate the drug release and antibacterial ability under different conditions. Moreover, the biocompatibility experiments and the in vivo full-thickness skin defect model results signify that the hydrogels have excellent biocompatibility and also can promote wound closure with accelerated wound contraction and improve the wound-healing process. All in all, the prepared temperature-sensitive nanocomposite hydrogels with improved mechanical properties, high biological safety, and accelerated wound healing have considerable practicability in the fields of drug delivery and wound dressing.
\end{abstract}

\section{Introduction}

Wound dressing refers to a type of material that can cover the surface of the skin wound to protect the wound temporarily and avoid infection. ${ }^{1-4}$ It can replace the skin in a short time and provide the wound with a low-oxygen environment to promote wound healing. In recent years, hydrogels have been proven to be a nearideal dressing to facilitate wound healing, which can keep the wound surface moist and isolate external microorganisms to prevent infection, and it can also fit the wound without adhesion to avoid secondary injury caused by dressing change. ${ }^{5,6}$ Smart hydrogel materials, ${ }^{7}$ a new-type dressing with huge potential in commercialization, respond to different external stimuli, resulting in a wider range of applications than traditional hydrogels. Undoubtedly, temperature-sensitive hydrogels ${ }^{8,9}$ are the most typical smart hydrogels. Poly( $N$-isopropylacrylamide) (PNIPAM) has been the most

\footnotetext{
${ }^{a}$ Guangdong Engineering and Technology Research Centre of Graphene-like Materials and Products, Department of Chemistry, College of Chemistry and Materials Science, Jinan University, Guangzhou 510632, China. E-mail: tsztan@jnu.edu.cn, thuanglh@jnu.edu.cn

${ }^{b}$ Guangdong Jianpai New Materials Co., Ltd., Foshan 528500, China
}

studied temperature-responsive polymer ${ }^{10,11}$ since its low critical solution temperature (LCST) behavior was first reported. However, studies have also shown that the PNIPAM hydrogels could release toxic amines ${ }^{12}$ in solution, greatly limiting its biological application. In contrast, the LCST behavior of poly- $N, N$-diethylacrylamide (PDEA) hydrogel $^{13}$ is similar to that of PNIPAM hydrogel. Note that the tertiary amide group will interact with the drugs containing hydrogen bonds when PEDA acts as a hydrogen bond acceptor, ${ }^{14,15}$ leading to the volume phase change of PEDA at LCST and drug release. Additionally, benefiting from the lower cytotoxicity and better biocompatibility than PNIPAM, PDEA has attracted more and more enthusiasm in the field of drug delivery and other fields in recent years. ${ }^{16,17}$

Unfortunately, hydrogels often encounter relatively weak mechanical strength owing to the formation of microporous morphology during the gelation process, ${ }^{18-20}$ which impedes its wide application in biomedical applications. In order to overcome these limitations, nanocomposite hydrogels have been developed ${ }^{21,22}$ by implanting various nano-materials into the network, which can not only improve mechanical properties, increase drug loading, and deliver drugs but also adjust and improve stimulus responsiveness. $^{23,24}$ Nanocomposite hydrogels were reported 
recently by adopting carbon nanotube (CNT) as the additive to enhance the mechanical property of the materials and to endow the hydrogels with excellent NIR stimuli-responsive ability. ${ }^{18,25-27}$ On the other hand, the rising incidence of infection by bacteria is another thorny issue in wound treatment. ${ }^{28-31}$ Meanwhile, wound dressings with inherent antibacterial ability represent better performance than broad-spectrum antibiotics in anti-wound infection. $^{28,32}$ Quaternized chitosan (QCS) not only exhibits more positive charges, which endow better antibacterial properties, ${ }^{33,34}$ but also exhibits better water solubility, hemostatic properties, and biocompatibility, ${ }^{35,36}$ suggesting that it has broader application prospects. $^{27,37}$ Thus, developing multifunctional CNT-enhanced QCS based temperature-sensitive nanocomposite hydrogels in wound dressings is highly desirable.

Based on this consideration, in this work, we propose the DEA-QCGM-CNT temperature-sensitive and NIR-sensitive multifunctional nanocomposite hydrogels with significantly improved mechanical properties. Particularly, the $\mathrm{DQ}_{0.5} \mathrm{C}_{1}$ hydrogel carrier with excellent performance in all aspects was selected and loaded with the antibacterial and anti-infective drug ciprofloxacin hydrochloride (CIP) to yield drug-loaded temperature-sensitive composite hydrogels with good biocompatibility. Wound healing experiments further proved that the prepared hydrogels exhibit the promoted ability of wound healing. In summary, all of the above results demonstrated the great potential application of DEA-QCGM-CNT hydrogels as a biomechanically active multifunctional hydrogel wound dressing for wound-healing, which will provide a new approach to design bioactive wound dressing.

\section{Experimental}

\subsection{Materials}

Carbon nanotubes (>97\%) were purchased from Nanoport Co., Ltd (Shenzhen, China). N,N-Diethylacrylamide (AR) was bought from Runjie Chemical Reagent Co., Ltd (Shanghai, China). Chitosan ( $\geq 98)$, concentrated nitric acid (98\%), and concentrated sulfuric acid (98\%) were purchased from Macklin Biochemical Technology Co., Ltd (Shanghai, China), and glycidyl trimethylammonium chloride $(80 \%)$ was obtained from Chemical Industry Co., Ltd (Tokyo). Glycidyl methacrylate (97\%), tetramethylethylenediamine (99\%), and ciprofloxacin hydrochloride $(98 \%)$, PBS $(\geq 98)$ were supplied by Aladdin Biochemical Technology Co., Ltd (Shanghai, China). Potassium persulfate (AR) and $N, N$-methylenebisacrylamide (AR) were obtained from Komiou Chemical Reagent Co., Ltd (Tianjin, China). Glacial acetic acid ( $\geq 98$ ) was bought from Guangzhou Chemical Reagent Factory.

\subsection{Synthesis of CNT}

CNT was synthesized by the purification of mixed acid. First, $0.5 \mathrm{~g}$ of raw carbon nanotubes (P-CNT) was weighed and added into a $100 \mathrm{~mL}$ three-necked flask, $10 \mathrm{~mL}$ of concentrated nitric acid and $30 \mathrm{~mL}$ of concentrated sulfuric acid were sequentially added with stirring, and then refluxed at $70{ }^{\circ} \mathrm{C}$ for $12 \mathrm{~h}$. After the reaction, the solution was diluted and washed with water by centrifugation. Finally, the products were dialyzed in deionized water for 5-7 days until the $\mathrm{pH}$ reached neutrality and freezedried for obtaining the CNT.

\subsection{Synthesis of QCGM}

The QCGM was synthesized via a one-pot reaction between epoxy groups from GTMAC and GMA and amino groups from CS. $1 \mathrm{~g}$ of CS was suspended in $36 \mathrm{~mL}$ of deionized water, and then $180 \mu \mathrm{L}$ of glacial acetic acid was added to the suspension. After stirring at $55{ }^{\circ} \mathrm{C}$ for $30 \mathrm{~min}, 1.84 \mathrm{~mL}$ of GTMAC was added dropwise to the mixed solution under continuous stirring. The reaction mixtures were stirred at $55{ }^{\circ} \mathrm{C}$ for $15 \mathrm{~h}$. Then, GMA was added dropwise to the above reaction mixtures with continuous stirring at $55{ }^{\circ} \mathrm{C}$, and the reaction was performed for another $15 \mathrm{~h}$ at $55{ }^{\circ} \mathrm{C}$ in dark conditions. After the reaction, the undissolved polymer was removed by centrifuging the mixture at $6000 \mathrm{rpm}$ for $20 \mathrm{~min}$ at room temperature. The supernatant liquid was precipitated into pre-cooled acetone to obtain the crude product. The crude product was further dissolved in deionized water and then dialyzed exhaustively against deionized water for seven days in dark conditions. Finally, the QCGM was obtained after freeze-drying. ${ }^{27}$ The quaternization degree of substitution (DS) ${ }^{32}$ of QCGM was determined by titrating the chlorine ion content.

\subsection{Preparation of DEA-QCGM-CNT temperature-sensitive hydrogels}

The hydrogels were prepared by polymerization of CNT aqueous dispersion and DEA/QCSG/N,N-methylenebisacrylamide (MBA) aqueous solution at room temperature using potassium persulfate (KPS)/tetramethylethylenediamine (TEMED) as redox initiator system. First, a certain amount of DEA monomer is dissolved in the QCGM and CNT aqueous solution, which has been ultrasonically dispersed in advance. After stirring for $30 \mathrm{~min}$, an appropriate amount of cross-linking agent MBA and $20 \mu \mathrm{L}$ of TEMED were added to the solution, and then the initiator potassium persulfate (KPS) was added after $2 \mathrm{~h}$ of ice bath ultrasonication. The reacted solution was quickly poured into a circular silica gel mold after the solution appeared flocculent and gelled at $25{ }^{\circ} \mathrm{C}$ for $24 \mathrm{~h}$. Finally, the molded hydrogel was released from the mold and placed in deionized water to soak for 3 days. In this work, the prepared hydrogels were denoted as $\mathrm{DQ}_{n} \mathrm{C}_{m}$, where the subscripts $n$ and $m$ represent the mass percentages of QCGM to DEA and CNT to DEA, respectively. The formulations for the synthesis of DEA-QCGMCNT temperature-sensitive hydrogels are listed in Table 1.

\subsection{Characterizations}

X-Ray diffraction (XRD, MSAL-XD2) was conducted to analyze the structures of $\mathrm{p}$-CNT and $\mathrm{CNT}$, which used a $\mathrm{Cu}-\mathrm{K} \alpha^{1}$ radia-

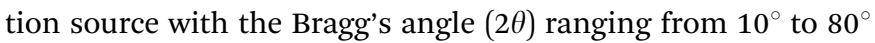
with the scan rate of $2^{\circ} \mathrm{min}^{-1}$. The spectra of CS and QCSG were obtained using a Bruker $500 \mathrm{MHz}$ NMR instrument with a small amount of CS and QCGM dissolved in 5\% (v/v) deuterated acetic acid/ $\mathrm{D}_{2} \mathrm{O}$ and $\mathrm{D}_{2} \mathrm{O}$, respectively. The spectra of the freeze- 
Table 1 The formulations for the synthesis of DEA-QCGM-CNT hydrogels

\begin{tabular}{llllll}
\hline Samples & $\begin{array}{l}\text { DEA } \\
(\mathrm{g})\end{array}$ & $\begin{array}{l}\text { QCGM:DEA } \\
(\mathrm{wt} \%)\end{array}$ & $\begin{array}{l}\text { CNT:DEA } \\
(\mathrm{wt} \%)\end{array}$ & $\begin{array}{l}\text { MBA:DEA } \\
(\mathrm{wt} \%)\end{array}$ & $\begin{array}{l}\text { KPS:DEA } \\
\text { (wt\%) }\end{array}$ \\
\hline $\mathrm{DQ}_{0} \mathrm{C}_{0}$ & 0.21 & 0 & 0 & 0.9 & 2.6 \\
$\mathrm{DQ}_{0.5} \mathrm{C}_{0}$ & 0.21 & 0.5 & 0 & 0.9 & 2.6 \\
$\mathrm{DQ}_{0.5} \mathrm{C}_{1}$ & 0.21 & 0.5 & 1 & 0.9 & 2.6 \\
$\mathrm{DQ}_{0.5} \mathrm{C}_{2}$ & 0.21 & 0.5 & 2 & 0.9 & 2.6 \\
$\mathrm{DQ}_{0} \mathrm{C}_{1}$ & 0.21 & 0 & 1 & 0.9 & 2.6 \\
$\mathrm{DQ}_{0.7} \mathrm{C}_{1}$ & 0.21 & 0.7 & 1 & 0.9 & 2.6 \\
\hline
\end{tabular}

dried p-CNT, CNT, CS, QCGM, and hydrogels were recorded in the range of $400-4000 \mathrm{~cm}^{-1}$ by employing a FT-IR spectrometer (Nicolet 6700). The morphologies of the freeze-dried hydrogels were observed using a field emission scanning electron microscope (FESEM, JEOLJEM-7600F). The thermal stability of CNT, QCGM, and hydrogels was analyzed by a thermogravimetric analyzer (TG, Germany Netzsch STA449F3) with a temperature range of $35-600{ }^{\circ} \mathrm{C}$, the heating rate of $10{ }^{\circ} \mathrm{C} \mathrm{min}{ }^{-1}$, and the $\mathrm{N}_{2}$

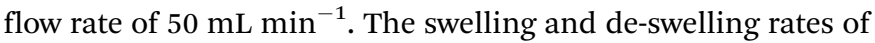
the temperature-sensitive hydrogels were determined using the gravimetric method, and then the swelling ratio $(\mathrm{SR})^{38}$ was calculated as: $\mathrm{SR}=\left(W_{t}-W_{d}\right)$, where the $W_{t}$ and $W_{\mathrm{d}}$ are the weights of the hydrogel at time $t$ and the weight of the dry hydrogel, respectively. Meanwhile, the de-swelling ratio (WL\%) was calculated as: $\mathrm{WL} \%=\left[\left(W_{\mathrm{e}}-W_{t}\right) /\left(W_{\mathrm{e}}-W_{\mathrm{d}}\right)\right] \times 100 \%$, where $W_{\mathrm{e}}$ is the weight of the equilibrium swelling hydrogels. A differential scanning calorimeter $(\mathrm{Q} 20)$ was used to test the LCST of the hydrogels. The equilibrium swelling rate (ESR) of the hydrogels was measured in the temperature range of 25 to $50{ }^{\circ} \mathrm{C}$, and the ESR was calculated as: ESR $=\left(W_{\mathrm{e}}-W_{\mathrm{d}}\right) / W_{\mathrm{d}}$ The mechanical behavior of as-prepared hydrogels was examined by performing tensile and compression tests using a universal testing machine (UTM-1422). The photothermal conversion performance of the as-prepared hydrogels was measured by NIR thermal imager. Briefly, the hydrogels were placed in a Petri dish containing deionized water and then irradiated with a NIR laser source at a power of $1.6 \mathrm{~W} \mathrm{~cm}^{-2}$ and a wavelength of $808 \mathrm{~nm}$ for $240 \mathrm{~s}$, and the temperature distribution of the hydrogels was recorded with NIR thermal imager.

\subsection{Drug loading and release in vitro}

CIP was used as a model drug to study the drug loading and release behavior of $\mathrm{DQ}_{0.5} \mathrm{C}_{1}$ hydrogel. For the preparation of drug-loaded hydrogels, the freeze-dried hydrogels were immersed in solutions with drug concentrations of 10, 20, and $50 \mu \mathrm{g} \mathrm{mL}^{-1}$ for $48 \mathrm{~h}$. The amount of CIP loaded into the hydrogels was determined using UV-vis spectroscopy. The drug loading of hydrogels ${ }^{5}$ was calculated as: $W_{\text {drug }}=\left(C_{0} V_{0}-C_{\mathrm{t}} V_{\mathrm{t}}\right) /$ $W_{\mathrm{d}}$, where the $C, C_{\mathrm{t}}, V_{0}$, and $V_{\mathrm{t}}$ are the initial drug loading concentration, the concentration of the remaining drug solution, the initial drug loading volume, and the remaining volume of the drug solution.

For release studies, the drug-loaded hydrogels were placed in PBS, and the solution was collected at predetermined time intervals and replaced with an equal amount of fresh PBS. The drug release behavior of $\mathrm{DQ}_{0.5} \mathrm{C}_{1}$ drug-loaded hydrogel at room temperature at 10,20 , and $50 \mu \mathrm{g} \mathrm{mL}^{-1}$ was investigated and the drug-loading concentrations of $20 \mu \mathrm{g} \mathrm{mL} \mathrm{m}^{-1}$ at $25{ }^{\circ} \mathrm{C}$ and $37{ }^{\circ} \mathrm{C}$ were also studied. The $\mathrm{DQ}_{0.5} \mathrm{C}_{1}$ hydrogel with a drug-loading concentration of $20 \mu \mathrm{g} \mathrm{mL} \mathrm{m}^{-1}$ at $25^{\circ} \mathrm{C}, 37^{\circ} \mathrm{C}$ and near infrared light ( $808 \mathrm{~nm}, 1.6 \mathrm{~W} \mathrm{~cm}^{-2}$ ) on and off cycles (on: $1 \mathrm{~min}$, off: $4 \mathrm{~min}$ ) were studied, respectively. The cumulative drug release ratio of hydrogels was calculated as: cumulative release $\%=$ $\left[\left(C_{\mathrm{ti}} \times V_{1}+V_{2} \times \Sigma C_{\mathrm{t}(i-1)}\right) / W_{\infty}\right] \times 100 \%$, where $C_{\mathrm{ti}}, W_{\infty}, V_{1}$, and $V_{2}$ are the drug release concentration at time $t$, the total amount of drug-loaded, the total volume, and the sampled volume.

\subsection{Antibacterial properties}

The method of inhibition zone, Gram-positive $S$. aureus (ATCC 6538) and Gram-negative E. coli (ATCC 8739) were applied to evaluate the antibacterial ability of the $\mathrm{DQ}_{0.5} \mathrm{C}_{1}$ drug-loaded hydrogel. Before antimicrobial assays, the logarithmic growth phase of bacteria was diluted with PBS until the optical density (OD) value at $600 \mathrm{~nm}$ was about $0.1-0.3$, where the bacterial concentration was nearly $10^{8}$ colonies $\left(\mathrm{CFU} \mathrm{mL}{ }^{-1}\right)$. In the subsequent steps, $10 \mathrm{~mL}$ of Agar was spread on a Petri dish, the oxford cup was added when the Agar was about to solidify, and then $10 \mathrm{~mL}$ of nutrient agar mixed with the bacterial solution evenly (the total number of colony was $10^{6} \mathrm{CFU} \mathrm{mL}{ }^{-1}$ ) was taken and covered on the Agar. Finally, the oxford cup was removed and the hydrogels were put in after the Agar solidified. The diameter of the inhibition zone was measured after incubation in a constant temperature incubator at $37{ }^{\circ} \mathrm{C}$ for $24 \mathrm{~h}$ to obtain the antibacterial activity. The antibacterial activities of $\mathrm{DQ}_{0.5} \mathrm{C}_{1}$ hydrogels with different drug loading concentrations against $E$. coli and $S$. aureus were tested.

\subsection{Bacteria live/dead test}

To observe the bacterial status visually, the bacterial live/dead measurement was performed. ${ }^{39}$ The bacteria $\left(1 \times 10^{8} \mathrm{CFU} \mathrm{mL}^{-1}\right)$ and the hydrogels were incubated together at $37^{\circ} \mathrm{C}$ for $12 \mathrm{~h}$, and the bacteria without any treatment were employed as the control group. Then, the physiological saline was used to wash and collect the bacteria, and live/dead stains (SYTO 9 and propidium iodide [PI]) were used to stain the bacteria for $15 \mathrm{~min}$ in the dark. After that, the stained bacterial solutions were then transferred to a confocal dish and observed using a confocal laser scanning microscope (ZEISS LSM880 with Airyscan, Germany).

\subsection{Hemolytic activity assay}

For hemolytic activity assay, $0.1 \mathrm{~g}$ hydrogel and a certain amount of rabbit fresh red blood cells ( $1 \%$ volume fraction) were added to a $2 \mathrm{~mL}$ centrifuge tube. Untreated and broken red blood cells with sterile water were used as negative and positive controls, respectively. The hydrogels with non-loaded $\operatorname{drug}\left(\mathrm{DQ}_{0.5} \mathrm{C}_{1}-0\right)$ and drug-loading concentration of $20 \mu \mathrm{g} \mathrm{mL}$ $\left(\mathrm{DQ}_{0.5} \mathrm{C}_{1}-20\right)$ were used as the experimental group. These solutions were incubated in an incubator at $37^{\circ} \mathrm{C}$ for $4 \mathrm{~h}$ and then centrifuged (3000 rpm, $3 \mathrm{~min}$ ) to obtain the supernatant. After that, the optical density of the supernatant was measured at a 
wavelength of $540 \mathrm{~nm}$, and the hemolysis ratio was calculated as:

$$
\text { Hemolysis }=\frac{\mathrm{OD}_{\text {sample }}-\mathrm{OD}_{\text {negative }}}{O D_{\text {positive }}-\mathrm{OD}_{\text {negative }}}
$$

$\mathrm{OD}_{\text {sample }}$, the optical density values of rabbit red blood cells of

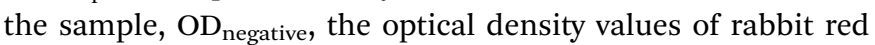
blood cells of the negative control, $\mathrm{OD}_{\text {positive, the optical density }}$ values of rabbit red blood cells of the positive control.

\subsection{Cytotoxicity test}

The cytotoxicity of human skin fibroblasts (HSF, purchased from Shanghai Cybertron Biotech) cultured with different concentrations of $\mathrm{DQ}_{0.5} \mathrm{C}_{1}$ hydrogel $\left(5,10,15,20\right.$, and $\left.30 \mathrm{mg} \mathrm{mL}^{-1}\right)$ was evaluated using the method of CCK-8, with untreated cells serving as the experimental control HSF cells in the logarithmic growth phase seeded into 96-well plates at $4 \times 10^{3}$ cells per well, cultured overnight in a constant temperature incubator at $37{ }^{\circ} \mathrm{C}$ with $5 \% \mathrm{CO}_{2}$. Then, the culture media containing different concentrations of hydrogel extracts were added to those wells for $24 \mathrm{~h}$ and then removed. Each well was washed three times with PBS where $100 \mu \mathrm{L}$ per well of $10 \%$ CCK- 8 medium was added to, and then it was cultured in a constant temperature incubator at $37{ }^{\circ} \mathrm{C}$ with $5 \% \mathrm{CO}_{2}$ for $2 \mathrm{~h}$. The microplate reader was used to measure the absorbance at $450 \mathrm{~nm}$ and calculated the relative cell viability. Finally, the live/dead kit was used to evaluate the survival of HSF cells. According to recommended procedures, after treatment of the live/dead kit, images were taken by a laser confocal microscope.

\subsection{In vivo wound healing test}

The wound healing ability of the hydrogels was investigated using six male Kun Ming (KM) rats (6-8 weeks, randomly divided into two groups equally). First, the rats were anesthetized with urethane anesthetic and the backs were depilated and locally disinfected with iodophor. Then, a round wound deep to the skin was cut without damaging the fascia and fatty tissue. The thickness of the wound was about $3 \mathrm{~mm}$ and the diameter was $7 \mathrm{~mm}$. The hydrogel was applied to the wound when the wound modeling was completed, and the dressing was changed on the 0 th, 3rd, 5 th, 7 th, 10 th, and 14 th day. The wound healing of the rats was recorded by the camera and
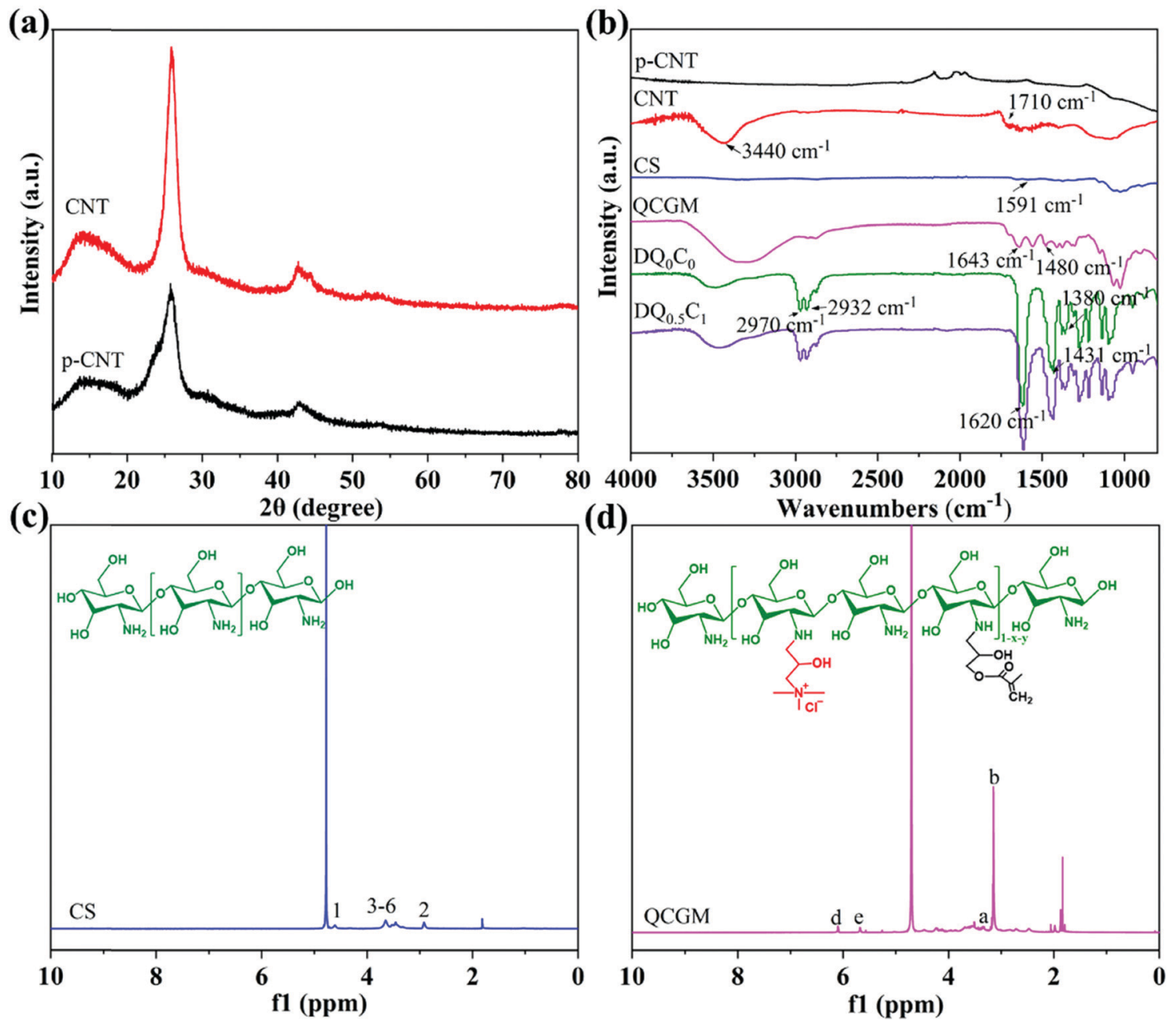

Fig. 1 XRD patterns (a) and of p-CNT and CNT and FT-IR spectra (b) of p-CNT, CNT, CS, QCGM, DQ ${ }_{0} C_{0}$ and $D Q_{0.5} C_{1}$ hydrogels, as well as ${ }^{1} H$ NMR spectra of CS (c) and QCGM (d). 
evaluated by the wound size. At the end of the experiment, the wound and the skin around the wound were harvested and immersed in $4 \%$ buffered paraformaldehyde. At last, the samples were stained with hematoxylin-eosin stain for histological observations. The Institutional Administration Panel for Laboratory Animal Care approved the experimental design. The university guidelines for care and use of laboratory animals were strictly followed. All animals were housed and fed in the Experimental Animal Center of Jinan University and were specific pathogen free.

\section{Results and discussion}

\subsection{Basic characterizations of DEA-QCGM-CNT temperature- sensitive hydrogels}

Shown in Fig. 1a is the XRD patterns of p-CNT and CNT. Diffraction peaks at $2 \theta$ of $25^{\circ}$ and $42^{\circ}$ correspond to the (002) and (100) crystal planes of p-CNT and CNT, ${ }^{40}$ respectively. It is worth noting that the diffraction peak at $2 \theta$ of $25^{\circ}$ of CNT is obviously enhanced, which is caused by the increase in the degree of graphitization of CNT due to the transformation of amorphous carbon. The infrared spectra of p-CNT, CNT, CS, QCGM, $\mathrm{DQ}_{0} \mathrm{C}_{0}$, and $\mathrm{DQ}_{0.5} \mathrm{C}_{1}$ hydrogels are illustrated in Fig. $1 \mathrm{~b}$. The absorption peak observed at 3440 and $1710 \mathrm{~cm}^{-1}$ of CNT belongs to the stretching vibration of $\mathrm{O}-\mathrm{H}$ and $\mathrm{C}=\mathrm{O}$, respectively, indicating that the carbon nanotubes have been oxidized by the mixed acid and attached with the carboxyl groups successfully. In the infrared spectrum of CS, the absorption peak at $1591 \mathrm{~cm}^{-1}$ is the deformation vibration peak of $-\mathrm{NH}_{2}$, while this peak in the spectrum of QCGM disappears accompanied with two new peaks appearing at 1480 and $1643 \mathrm{~cm}^{-1}$, which are attributed to the $\mathrm{C}-\mathrm{H}$ of methyl on GTMAC and the $\mathrm{C}=\mathrm{C}$ stretching vibration peak on GMA, respectively. In addition, the stretching vibration peaks at $1027 \mathrm{~cm}^{-1}$ of $\mathrm{C}-\mathrm{OH}$ and $1034 \mathrm{~cm}^{-1}$ of $\mathrm{C}-\mathrm{O}$ on the CS spectrum can also be observed in the QCGM, indicating that QCGM have been successfully synthesized and the substitution mainly occurred on the amino group of CS. A similar result can also be verified in the ${ }^{1} \mathrm{H}$ NMR spectra of CS and QCGM (Fig. 1c and d). The hydrogen absorption peaks of CS shown in Fig. 1c are consistent with the ref. 41 . In the spectrum of QCGM, the peak at $\delta \approx 4.65 \mathrm{ppm}$ was the peak of $\mathrm{D}_{2} \mathrm{O}$, and the peak at $\delta \approx 3.15 \mathrm{ppm}$ is attributed to the proton peaks $(\mathrm{H}-\mathrm{b})$ on the three methyl groups in the $-\mathrm{N}^{+}\left(\mathrm{CH}_{3}\right)_{3}$ group. Especially, the peak at $\delta \approx 3.32 \mathrm{ppm}$ corresponded to the proton peak $(\mathrm{H}-\mathrm{a})^{42}$ on the $-\mathrm{N}-\mathrm{CH}_{2}-$ group. At the same time, two peaks of $\delta \approx 5.66 \mathrm{ppm}(\mathrm{H}-\mathrm{c})$ and $6.07 \mathrm{ppm}(\mathrm{H}-\mathrm{d})$ appear in QCGM, which correspond to the proton peaks on the GMA double bond, respectively, proving that CS is successfully grafted with GMA and GTMAC. Additionally, the peaks at 2970 and $2932 \mathrm{~cm}^{-1}$ observed in the infrared spectrum of the $\mathrm{DQ}_{0} \mathrm{C}_{0}$ hydrogel are attributed to the $\mathrm{C}-\mathrm{H}$ stretching vibration absorption peaks of $-\mathrm{CH}_{3}$ and $-\mathrm{CH}_{2}$ groups. The absorption peak at $1620 \mathrm{~cm}^{-1}$ belongs to the $\mathrm{C}=\mathrm{O}$ bond in the amide and the peaks at 1431 and $1380 \mathrm{~cm}^{-1}$ represent the symmetrical $\mathrm{C}-\mathrm{H}$ in-plane bending vibration
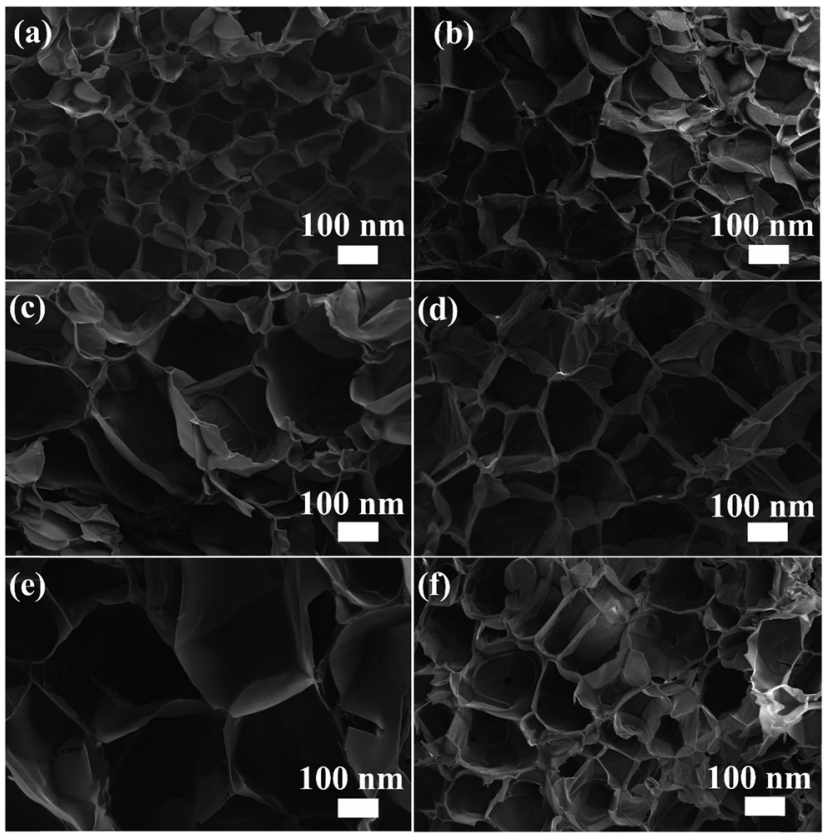

Fig. 2 SEM images of $D Q_{0} C_{0}$ (a), $D Q_{0.5} C_{0}$ (b), $D Q_{0.5} C_{1}$ (c), $D Q_{0.5} C_{2}$ (d), $D Q_{0} C_{1}(e)$, and $D Q_{0.7} C_{1}(f)$ hydrogels.

absorption peak. ${ }^{43}$ Importantly, the above absorption peaks all appear in the infrared spectrum of the $\mathrm{DQ}_{0.5} \mathrm{C}_{1}$ temperaturesensitive hydrogel except for some differences in peak intensity, which proves that the DEA-QCGM-CNT series of temperaturesensitive hydrogels have been successfully synthesized.

The microstructures of DEA-QCGM-CNT temperaturesensitive hydrogels are observed by FESEM, with the results shown in Fig. 2. All hydrogels exhibit a honeycomb structure, and the pore sizes of the hydrogels are enlarged with the addition of CNT and QCGM when compared to the $\mathrm{DQ}_{0} \mathrm{C}_{0}$ hydrogel. With the increase of CNT, the pore size of the hydrogels increases first and then decreases when the QCGM

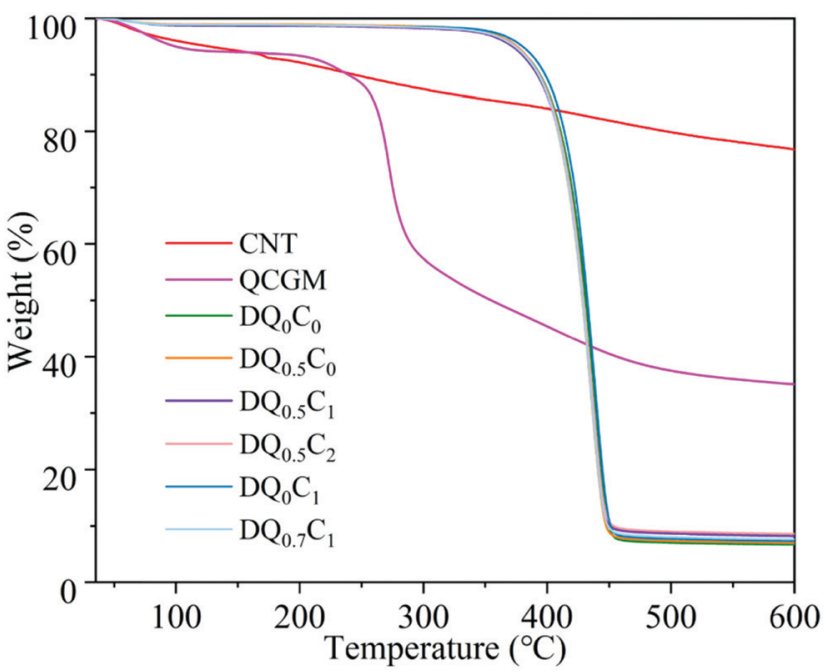

Fig. 3 Thermal decomposition curves of CNT, QCGM, and DEA-QCGMCNT hydrogels. 
(a)
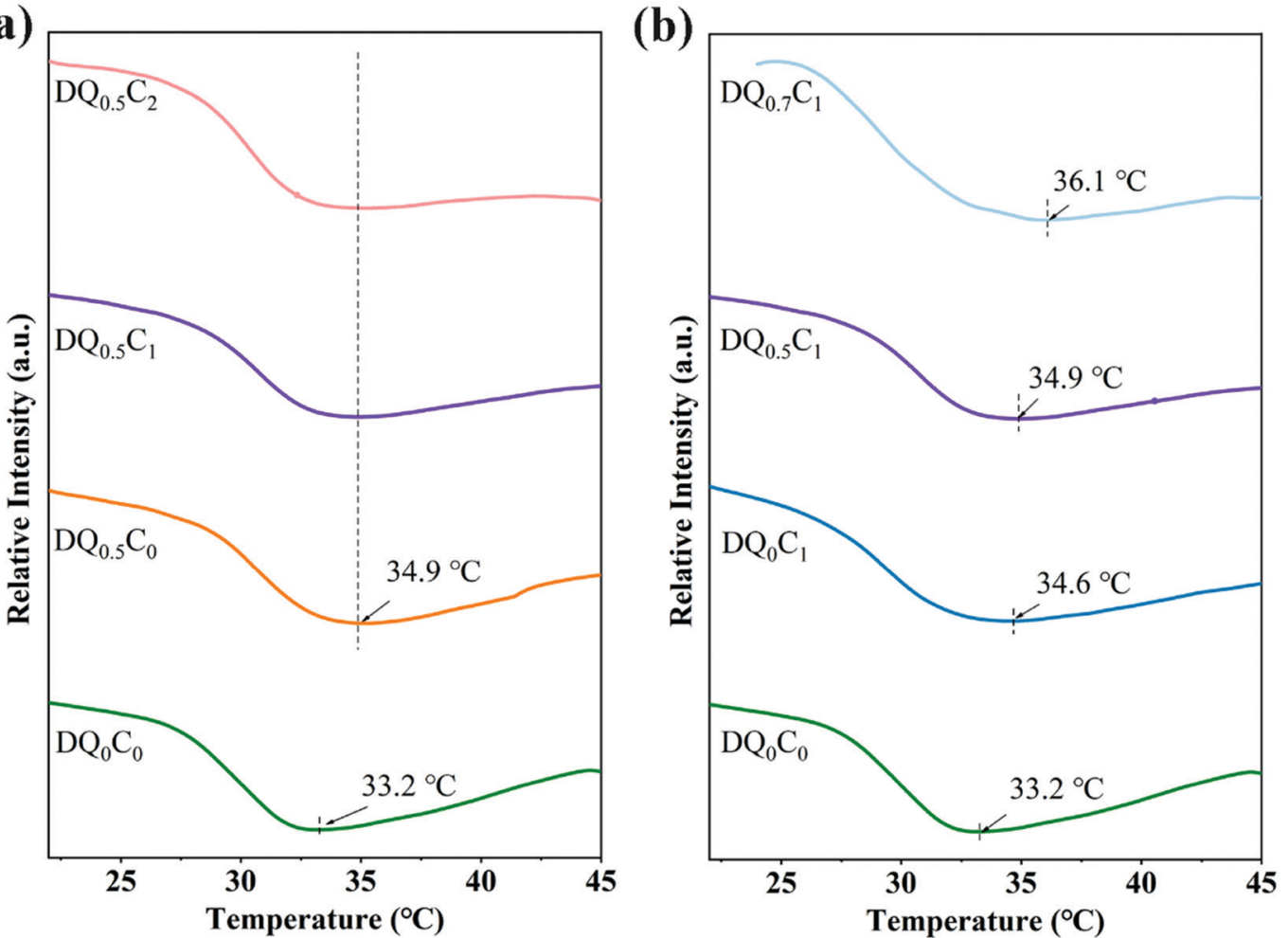

Fig. 4 The DSC curves of DEA-QCGM-CNT hydrogels with different CNT contents (a) and different QCGM contents (b).

remains unchanged (Fig. 2b-d). The reason is that CNT contains a large number of hydrophilic groups such as hydroxyl and carboxyl groups, which can attract more water into the hydrogels, resulting in larger water content and voids. Whereas CNT will accumulate in the hydrogels when the content exceeds a certain value, causing uneven cross-linking and a decrease in pore size within the hydrogels. ${ }^{44}$ In addition, the pore size gradually decreases with the increase of QCGM while keeping the CNT addition amount constant. This was owing to the double bond contained in QCGM. As the content of QCGM increases, more QCGM will be introduced into the system for cross-linking. The increase of chain length, effective crosslinking, and cross-linking density in hydrogels are responsible for the smaller pore size.

In order to explore the influence of CNT and QCGM on the thermal stability of hydrogels, CNT, QCGM, and DEA-QCGMCNT temperature-sensitive hydrogels were subjected to TG tests (Fig. 3). The mass loss of CNT before $130{ }^{\circ} \mathrm{C}$ is about $6 \mathrm{wt} \%$, corresponding to the evaporation of adsorbed water, and the mass loss of CNT in the range of $150-200{ }^{\circ} \mathrm{C}$ was $5 \mathrm{wt} \%$, which was mainly caused by the decarboxylation of carboxyl groups on CNT. ${ }^{45}$ CNT behaves very stably throughout the heating process, with only $23 \mathrm{wt} \%$ mass loss even when heated to $600{ }^{\circ} \mathrm{C}$. Compared with CNT, QCGM shows less mass loss before $125{ }^{\circ} \mathrm{C}$, but $45 \mathrm{wt} \%$ mass loss occurs in the temperature range of $185-361{ }^{\circ} \mathrm{C}$, which was attributed to the degradation of the main chain of QCGM. Similarly, the thermal decomposition of the hydrogels can be divided into two stages. The first stage is in the range of $46-95{ }^{\circ} \mathrm{C}$ with less mass loss, corresponding to the evaporation of water in the hydrogels. The mass loss during the stage at $300-470{ }^{\circ} \mathrm{C}$ is about $90 \mathrm{wt} \%$, which is caused by the degradation of the polymer backbone or the breakage of the polymer side groups. ${ }^{46}$ Note that no significant difference is found in the thermogravimetric curves of the six groups of hydrogels, which is mainly ascribed to the tiny addition of CNT and QCGM.

It is well-known that there exists a tradeoff between affinity and hydrophobicity in the molecular chain of DEA-QCGM-CNT thermosensitive hydrogels. When the external temperature is lower than the LCST, the hydrophilic interaction between the hydrophilic groups (carboxyl, amide, and hydroxyl) in the hydrogel and the water molecules will increase, resulting in the hydrogels showing a swelling state. Conversely, the hydrogen bond between the hydrophilic group and the water molecule will be weakened, and the hydrophobic interaction between the molecular chains will be strengthened when the external temperature is higher than LCST, causing the shrinkage and entanglement of the molecular chains so that the hydrogels assume a contracted state. This process will also be accompanied by thermal effects, so the thermal behavior of the hydrogels was studied by DSC. The DSC curves of DEA-QCGMCNT temperature-sensitive hydrogels are shown in Fig. 4. It can be found that the addition of CNT could increase the LCST by comparing it with the $\mathrm{DQ}_{0} \mathrm{C}_{0}$ and $\mathrm{DQ}_{0} \mathrm{C}_{1}$ hydrogels. However, the LCST of $\mathrm{DQ}_{0.5} \mathrm{C}_{0}, \mathrm{DQ}_{0.5} \mathrm{C}_{1}$, and $\mathrm{DQ}_{0.5} \mathrm{C}_{2}$ hydrogels (Fig. 4a) are around $34.9{ }^{\circ} \mathrm{C}$, which did not change with the content of CNTs basically when the amount of QCGM remained constant. Moreover, when CNT remained unchanged (Fig. 4b), the LCST 
(a)
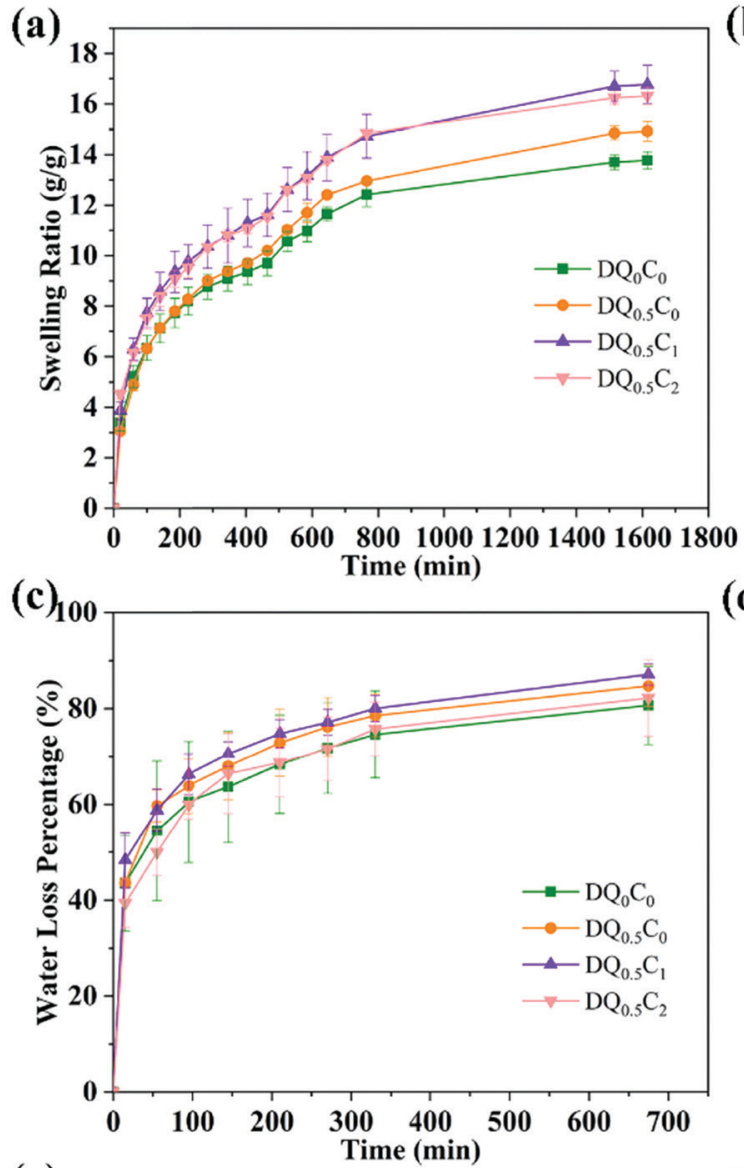

(e)

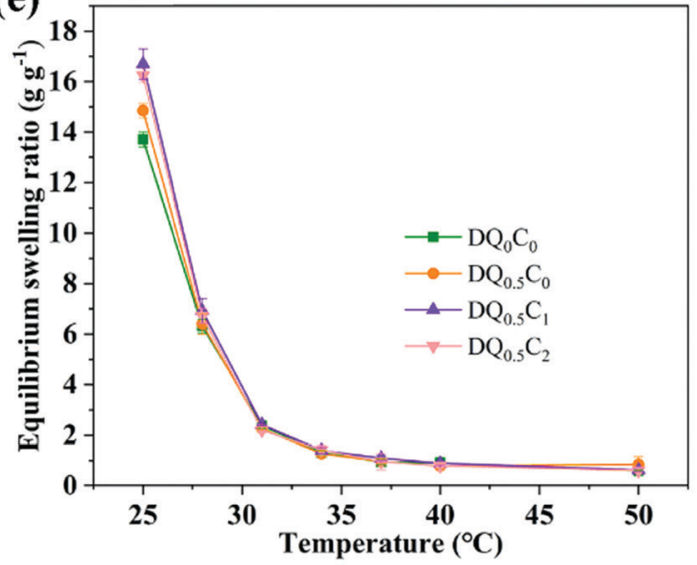

(b)

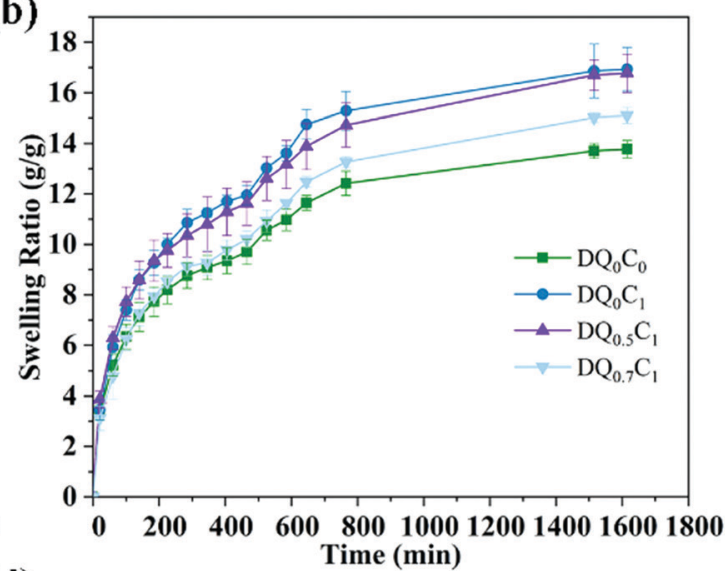

(d)

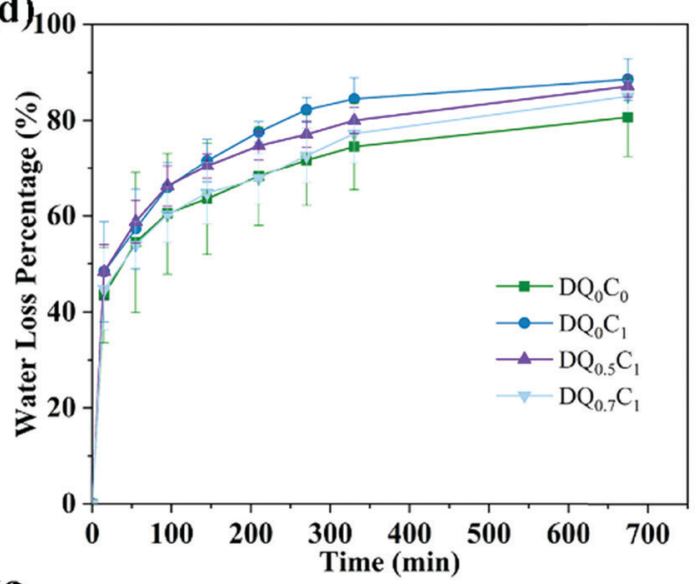

(f)

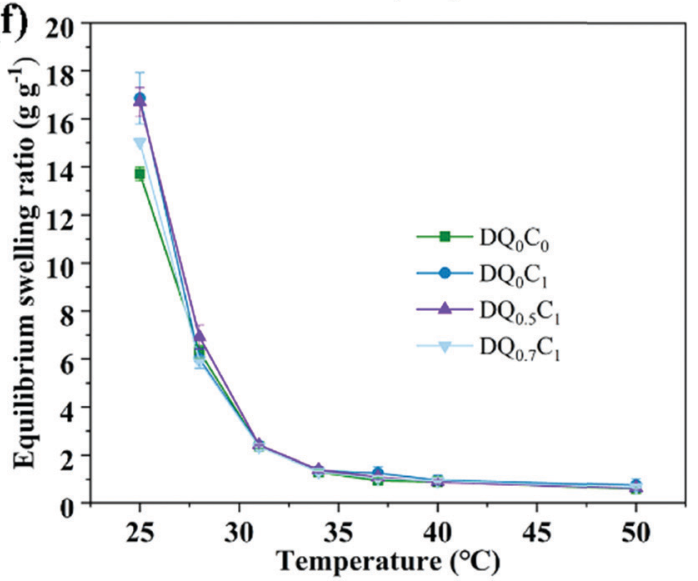

Fig. 5 Swelling rate curves ( $a$ and b) of DEA-QCGM-CNT hydrogels, de-swelling rate curves ( $c$ and d) of DEA-QCGM-CNT hydrogels, and the equilibrium swelling ratio (e and f) of DEA-QCGM-CNT hydrogels with temperature.

of the hydrogels increased with the addition of the QCGM, and all of them were higher than that of the $\mathrm{DQ}_{0} \mathrm{C}_{0}$ hydrogel. The reason for the above results is that the addition of QCGM introduces more hydrophilic groups (hydroxyl, amino, etc.) into the hydrogels, which promotes the formation of hydrogen bonds and strengthens the binding force between molecules. Therefore, more energy is required for the hydrogels to undergo a phase transition, thus leading to the elevation of LCST. $^{44}$ Meaningfully, the LCST of the PDEA hydrogels can be controlled by adjusting the amount of QCGM.
The swelling ratio, de-swelling ratio, and equilibrium swelling ratio are important parameters for revealing the interaction between the hydrogels and the polymer chains. First, the swelling ratio of DEA-QCGM-CNT hydrogels in deionized water at $25{ }^{\circ} \mathrm{C}$ was explored, with the results shown in Fig. 5a and $\mathrm{b}$. It can be found that the swelling ratio of all hydrogels increased with time and reached the maximum after $25 \mathrm{~h}$ and remained in equilibrium. Given that the internal pores will be saturated completely when the hydrogels reach the maximum swelling ratio, the swelling ratio thus no longer changes with 
the swelling time. ${ }^{46}$ The swelling ratios of $\mathrm{DQ}_{0.5} \mathrm{C}_{0}, \mathrm{DQ}_{0.5} \mathrm{C}_{1}$, $\mathrm{DQ}_{0.5} \mathrm{C}_{2}, \mathrm{DQ}_{0} \mathrm{C}_{1}$, and $\mathrm{DQ}_{0.7} \mathrm{C}_{1}$ hydrogels after $25 \mathrm{~h}$ are 14.92, $16.77,16.32$, 16.93, and $15.10 \mathrm{~g} \mathrm{~g}^{-1}$, respectively, which are all higher than $\mathrm{DQ}_{0} \mathrm{C}_{0}$ hydrogel without QCGM and CNT $\left(13.77 \mathrm{~g} \mathrm{~g}^{-1}\right)$. The enhanced swelling ratio is expected since mainly QCGM contains $-\mathrm{OH},-\mathrm{NH}_{2}$ and CNT contains $-\mathrm{OH}$, $-\mathrm{COOH}$, and other hydrophilic groups, which can attract water molecules from the surrounding environment into the hydrogels to increase the pore size. ${ }^{47}$ Additionally, the swelling ratio varies with the content of QCGM and CNT. As shown in Fig. 5a, keeping the addition of QCGM constant, the swelling ratio exhibits a trend of first increasing and then decreasing with the increase of CNT, which is consistent with the changes in the pore size of the hydrogels observed in FESEM. At the same time, the swelling ratio with more QCGM was lower when keeping the CNT constant. Owing to the decreasing pore size of the hydrogels with the increase of QCGM, the weaker water absorption capacity and decreased swelling rate are responsible for the lower swelling ratio with more QCGM.

Secondly, the de-swelling ability of DEA-QCGM-CNT temperature-sensitive hydrogels in deionized water at $45{ }^{\circ} \mathrm{C}$ was explored and the results are presented in Fig. $5 \mathrm{c}$ and d. All hydrogels lost water immediately after being placed in deionized water at $45{ }^{\circ} \mathrm{C}$, where $\mathrm{DQ}_{0} \mathrm{C}_{0}$ hydrogels enable the lowest de-swelling and response speed. The de-swelling ratios of $\mathrm{DQ}_{0.5} \mathrm{C}_{0}, \mathrm{DQ}_{0.5} \mathrm{C}_{1}, \mathrm{DQ}_{0.5} \mathrm{C}_{2}, \mathrm{DQ}_{0} \mathrm{C}_{1}$, and $\mathrm{DQ}_{0.7} \mathrm{C}_{1}$ hydrogels are $84.70 \%, 87.09 \%, 82.18 \%, 88.55 \%$, and $85.01 \%$, respectively. However, the de-swelling ratios of $\mathrm{DQ}_{0} \mathrm{C}_{0}$ hydrogel is $80.64 \%$ within $675 \mathrm{~min}$, which is caused by the stronger interaction between the hydrogels and water molecules with the addition of CNT and QCGM.

Finally, the temperature sensitivity was tested by measuring the equilibrium swelling ratio of DEA-QCGM-CNT hydrogels with the variation of temperature. As shown in Fig. 5e and $f$, the equilibrium swelling ratio of DEA-QCGM-CNT hydrogels decreased with increasing temperature, suggesting that they are typical heat-shrinkable temperature-sensitive hydrogels. This is due to the presence of hydrophilic groups $(-\mathrm{OH}$, $\left.-\mathrm{COOH},-\mathrm{CONR}_{2}\right)$ and hydrophobic groups $\left(-\mathrm{CH}_{2} \mathrm{CH}_{3}\right)$ in the hydrogels. Thereinto, the hydrophilic groups in the hydrogels at lower temperatures combine with water molecules through hydrogen bonds to make the polymer chains in a stretched state, leading the hydrogels to absorb water and swell. On the contrary, owing to the accelerated movement of water molecules, the hydrogen bonding will be weakened, the force of the hydrophobic groups of the polymer chains in the hydrogels will
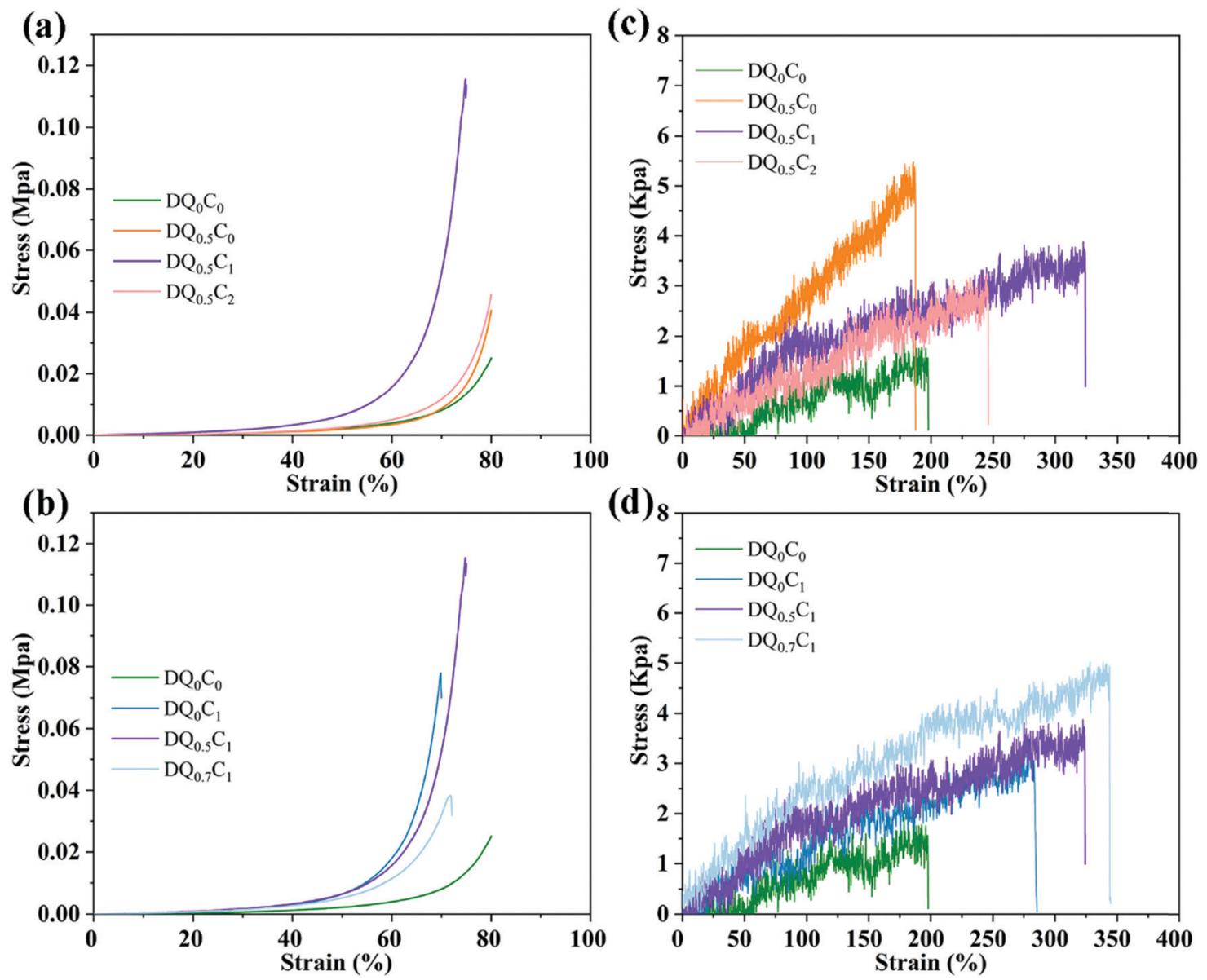

Fig. 6 Compressive stress-strain curves of hydrogels with different contents of CNT (a) and QCGM (b), respectively, and tensile stress-strain curves of hydrogels with different contents of CNT (c) and QCGM (d), respectively. 
be strengthened, and the structure of the hydrogels will become denser as well as when the temperature rises, which causes water molecules to be squeezed out and decrease the equilibrium swelling ratio. In a word, the investigation of swelling ratio, de-swelling rate, and equilibrium swelling ratio of DEAQCGM-CNT hydrogels demonstrates that proper introduction of QCGM and CNT to the PDEA hydrogels benefits the swelling and response capability of the hydrogels.

Excellent mechanical properties are of prime importance for the practical application of hydrogels in the field of drug carriers. Therefore, the compression and tensile properties of DEA-QCGM-CNT hydrogels were evaluated, with the results displayed in Fig. 6. The compressive stress of all hydrogels increases tardily with the increase of strain when the strain is less than $40 \%$ (Fig. 6a and b). The hydrogels are in an elastic state at this stage and return easily to the original shape once the compressive stress is removed. Conversely, when the strain of the hydrogel increased to $40-80 \%$, the compressive stress increased rapidly, indicating that energy dissipation occurred inside the hydrogels to resist the compressive stress. ${ }^{48}$ As shown in Fig. 6a, the maximum compressive strengths of $\mathrm{DQ}_{0} \mathrm{C}_{0}, \mathrm{DQ}_{0.5} \mathrm{C}_{0}, \mathrm{DQ}_{0.5} \mathrm{C}_{1}$, and $\mathrm{DQ}_{0.5} \mathrm{C}_{2}$ are $0.025,0.040,0.116$, and $0.046 \mathrm{MPa}$, respectively. When the content of QCGM remains unchanged, the compressive strength of the hydrogels increases first and then decreases with the increasing content of CNT. This is due to the fact that a small amount of CNTs can be uniformly dispersed in the hydrogels and cross-linked with the hydrogels through hydrogen bonds, which will increase the cross-linking density and compressive load capacity. However, CNTs will aggregate in the hydrogels when the added amount is too much, resulting in uneven cross-linking and decreased compressive strength. Additionally, the influence of QCGM on
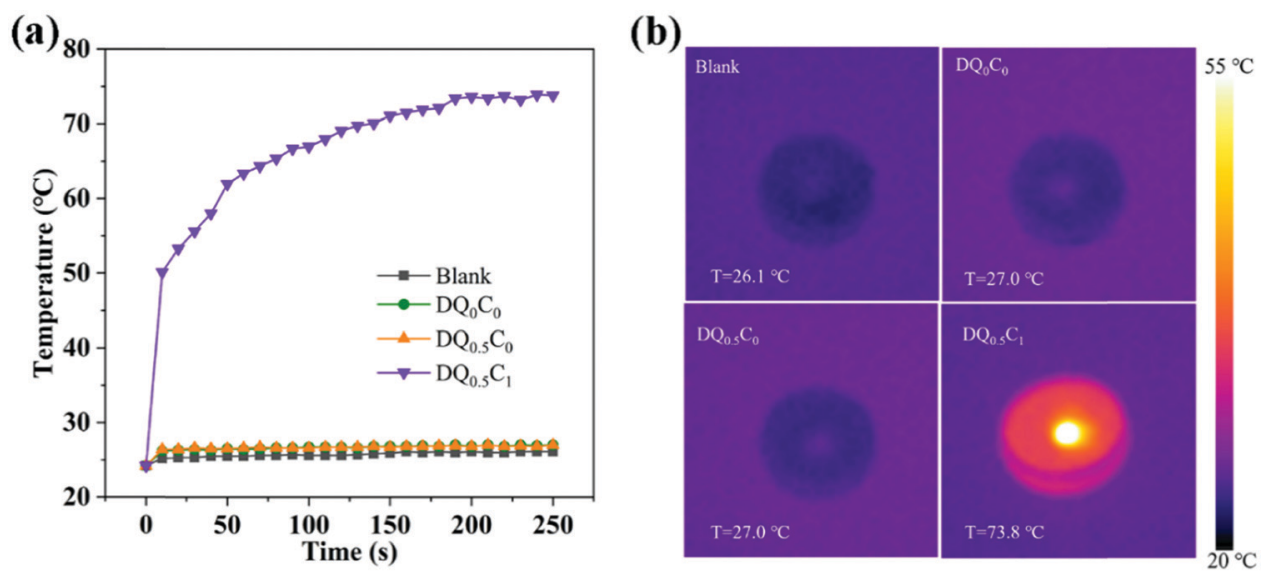

(c)

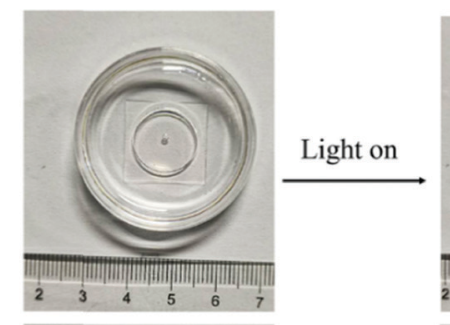

(d)
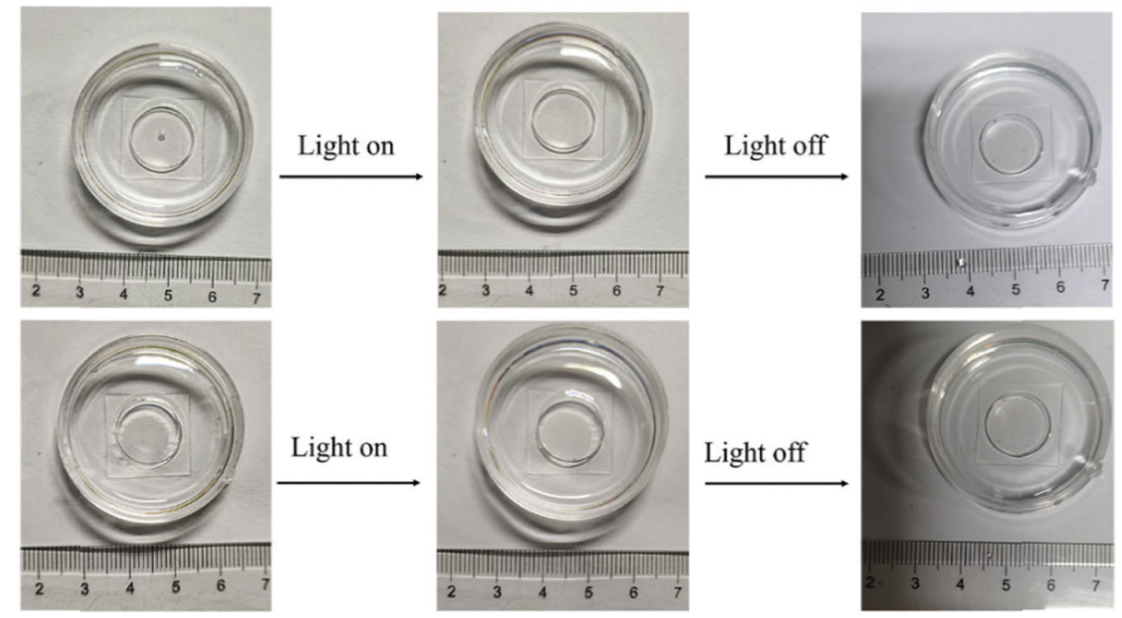

(e)
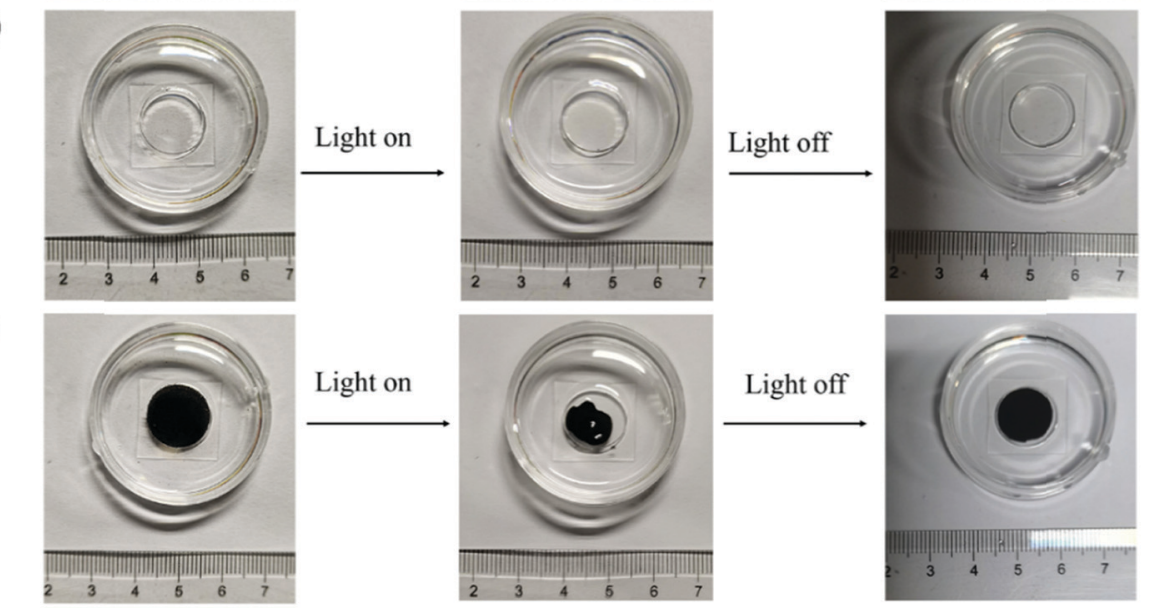

Fig. 7 The temperature changes (a) and thermal images (b) of deionized water (Blank), $D Q_{0} C_{0}, D_{0.5} C_{0}$, and $D Q_{0.5} C_{1}$ hydrogels under $N I R$ light irradiation as well as the shape changes of $D Q_{0} C_{0}$ (c), $D Q_{0.5} C_{0}$ (d), and $D Q_{0.5} C_{1}$ (e) hydrogels before and after NIR light irradiation. 
the compression performance of the hydrogels when the CNT amount remains constant is also explored as shown in Fig. $6 \mathrm{~b}$. $\mathrm{DQ}_{0.5} \mathrm{C}_{1}$ hydrogel exhibits the best compression performance when it reaches the maximum compression deformation, which is $0.116 \mathrm{MPa}\left(\mathrm{DQ}_{0} \mathrm{C}_{1}\right.$ hydrogel was only $\left.0.078 \mathrm{MPa}\right)$, proving that the addition of QCGM could improve the compressive strength of the hydrogels.

The influence of CNT on the tensile properties of the hydrogels was explored by keeping the content of QCGM unchanged, as shown in Fig. 6c. The tensile strengths of $\mathrm{DQ}_{0.5} \mathrm{C}_{0}, \mathrm{DQ}_{0.5} \mathrm{C}_{1}$, and $\mathrm{DQ}_{0.5} \mathrm{C}_{2}$ hydrogels are 5.32, 3.73, and $3.23 \mathrm{kPa}$, respectively, and the tensile strengths of $\mathrm{DQ}_{0} \mathrm{C}_{1}$, $\mathrm{DQ}_{0.5} \mathrm{C}_{1}$, and $\mathrm{DQ}_{0.7} \mathrm{C}_{1}$ hydrogels are $3.09,3.73$, and $4.90 \mathrm{kPa}$ (all higher than $\mathrm{DQ}_{0} \mathrm{C}_{0}$ hydrogel), respectively. It indicates that the tensile strength of the hydrogels decreased gradually with the increase of CNT and increased gradually with the increase of QCGM. That is to say, the addition of QCGM and CNT can greatly improve the mechanical properties of PDEA hydrogels and $\mathrm{DQ}_{0.5} \mathrm{C}_{1}$ hydrogels exhibits superior mechanical properties than other hydrogels. Therefore, the $\mathrm{DQ}_{0.5} \mathrm{C}_{1}$ hydrogel with outstanding swelling and mechanical properties was subsequently selected and loaded with the antibacterial model drug CIP for investigating the photothermal conversion performance, drug loading and release, antibacterial performance, biocompatibility, and the application of wound dressings.

\subsection{Photothermal conversion properties of hydrogels}

The photothermal conversion ability of the hydrogels is evaluated using a thermal imager to record temperature changes of the deionized water (Blank), $\mathrm{DQ}_{0} \mathrm{C}_{0}, \mathrm{DQ}_{0.5} \mathrm{C}_{0}$, and $\mathrm{DQ}_{0.5} \mathrm{C}_{1}$ under $808 \mathrm{~nm}$ and $1.6 \mathrm{~W} \mathrm{~cm}^{-2}$ NIR light irradiation, and the results are shown in Fig. 7. The temperature changes of Blank, $\mathrm{DQ}_{0} \mathrm{C}_{0}$, and $\mathrm{DQ}_{0.5} \mathrm{C}_{0}$ hydrogels are not obvious near room temperature, while the temperature of $\mathrm{DQ}_{0.5} \mathrm{C}_{1}$ hydrogel changes significantly under the same conditions with the temperature increasing from $24.2{ }^{\circ} \mathrm{C}$ to $73.8{ }^{\circ} \mathrm{C}$ after $250 \mathrm{~s}$ of NIR light irradiation (Fig. 7a and b). In addition, despite the fact that $\mathrm{DQ}_{0} \mathrm{C}_{0}$ and $\mathrm{DQ}_{0.5} \mathrm{C}_{0}$ hydrogels do not contain CNTs, they still maintained their original shape after $250 \mathrm{~s}$ of NIR light irradiation (Fig. $7 \mathrm{c}$ and $\mathrm{d}$ ). Interestingly, the $\mathrm{DQ}_{0.5} \mathrm{C}_{1}$ hydrogel shrinks tremendously and is restored after turning off the NIR light for a while (Fig. 7e). All of the above analyses indicate that CNT can endow the hydrogels with excellent photothermal conversion ability.
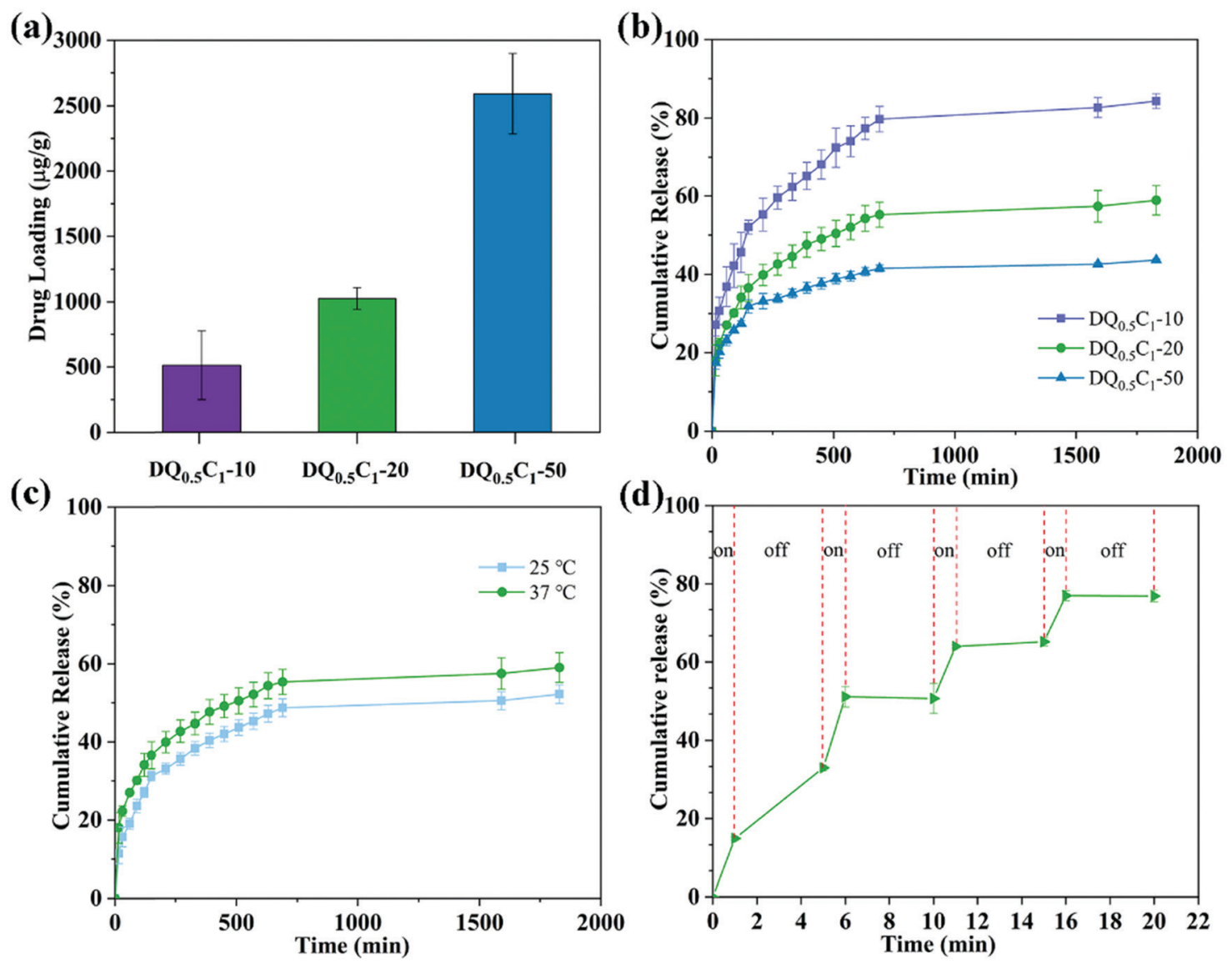

Fig. 8 (a) Drug loading capacity of $\mathrm{DQ}_{0.5} \mathrm{C}_{1}$ hydrogel in different concentrations; (b) drug release curve of hydrogel with different drug loadings at $37{ }^{\circ} \mathrm{C}$; (c) drug release behavior of $D Q_{0.5} C_{1}-20$ hydrogel at different temperatures; (d) NIR controlled CIP cumulative release of $D Q_{0.5} C_{1}-20$ hydrogel. 


\subsection{Drug loading and release}

The moist and warm environment of the wound surface is very suitable for the growth and reproduction of bacteria, which will make the wound extremely vulnerable to infection and hinder wound healing. Therefore, it is necessary for the hydrogels used in the biomedical field to possess certain antibacterial properties. The drug loading capacity of $\mathrm{DQ}_{0.5} \mathrm{C}_{1}$ hydrogels in different concentrations of CIP is shown in Fig. 8a, where the UV-vis spectrometer is applied to measure the concentrations of CIP in the solution before and after drug loading at $276 \mathrm{~nm}$. Clearly, when the concentrations of CIP are 10, 20, and $50 \mu \mathrm{g} \mathrm{mL}{ }^{-1}$, the drug loading contents reach 510.72, 1023.35, and $2591.99 \mu \mathrm{g} \mathrm{g}^{-1}$, respectively. The drug loading will inevitably increase when the concentration of the drug increases because the drug loading of the hydrogels is affected by the swelling ratio.

Shown in Fig. $8 \mathrm{~b}$ are the cumulative drug release curves of $\mathrm{DQ}_{0.5} \mathrm{C}_{1}$ hydrogels with different drug loading concentrations at $37^{\circ} \mathrm{C}$. In the first $150 \mathrm{~min}$, the rapid drug release is exhibited by almost in a linear manner, while roughly unchanged and tend to be in equilibrium at about $1600 \mathrm{~min}$. The cumulative drug release rates of $\mathrm{DQ}_{0.5} \mathrm{C}_{1}-10, \mathrm{DQ}_{0.5} \mathrm{C}_{1}-20$, and $\mathrm{DQ}_{0.5} \mathrm{C}_{1}-50$ were $84.36 \%, 58.89 \%$, and $43.70 \%$ when they reached equilibrium, respectively, indicating downward trends as the drug concentration increased. The carboxyl group on CIP can easily form a hydrogen bond with the tertiary amide on the DEA chain, resulting in a decrease in the cumulative release of the drug.

The drug release behavior of $\mathrm{DQ}_{0.5} \mathrm{C}_{1}-20$ hydrogels at different temperatures is presented in Fig. 8c. The cumulative drug release ratios of the hydrogels gradually increased with the increase of temperature, and it reached $52.07 \%$ and $58.89 \%$ at $25{ }^{\circ} \mathrm{C}$ and $37{ }^{\circ} \mathrm{C}$ when the release approached the balance state, respectively. The hydrogels displayed a swelling state at $25{ }^{\circ} \mathrm{C}$ $(<\mathrm{LCST})$ and the drug is only released by generating the difference in concentration between the inside and outside of the hydrogel at the same time. However, the hydrogels are hydrophobic and shrunk at $37{ }^{\circ} \mathrm{C}$ (> LCST), and thus the drug is released through the dual effects of the shrinkage and extrusion of the hydrogels and the difference in internal and external concentrations, resulting in a higher release ratio than at $25{ }^{\circ} \mathrm{C}$.

Interestingly, $\mathrm{DQ}_{0.5} \mathrm{C}_{1}-20$ hydrogels exhibited excellent NIR controllability for the release of CIP. As shown in Fig. 8d, NIR light irradiation can significantly improve the drug release ratio. After free diffusion (the first $5 \mathrm{~min}$ ), the drug is released when irradiated with NIR light for $1 \mathrm{~min}$ and no further release is observed after turning off the NIR light. On the other hand, under NIR light irradiation, the released drug amount of $\mathrm{DQ}_{0.5} \mathrm{C}_{1}-20$ hydrogels increases significantly, and the cumulative drug release ratio reaches $76.94 \%$ within $20 \mathrm{~min}$, which is significantly higher than that without NIR light irradiation at the same temperature. The above results illustrate that the CIP molecules loaded on the hydrogels can be released through the "on-off" mode triggered by the NIR light, meaning that the drug release ability of the hydrogels could be effectively controlled by NIR light. This controllable drug release method is considered to be beneficial for prolonging the accumulation of drugs at the infected site and treating bacterial infections accurately on demand.

Table 2 The diameter of inhibition zones of $\mathrm{DQ}_{0.5} \mathrm{C}_{1}$ hydrogel with different drug loading concentrations

\begin{tabular}{lll}
\hline & \multicolumn{2}{l}{ Diameter of the inhibition zone $(\mathrm{mm})$} \\
\cline { 2 - 3 } Samples & E. coli & S. aureus \\
\hline DQ $_{0.5} C_{1}-0$ & 0 & 0 \\
DQ $_{0.5} C_{1}-10$ & $24.47 \pm 0.55$ & $16.33 \pm 0.58$ \\
DQ $_{0.5} C_{1}-20$ & $26.17 \pm 0.29$ & $21.67 \pm 0.39$ \\
DQ $_{0.5} C_{1}-50$ & $29.67 \pm 0.76$ & $25.00 \pm 0.50$
\end{tabular}

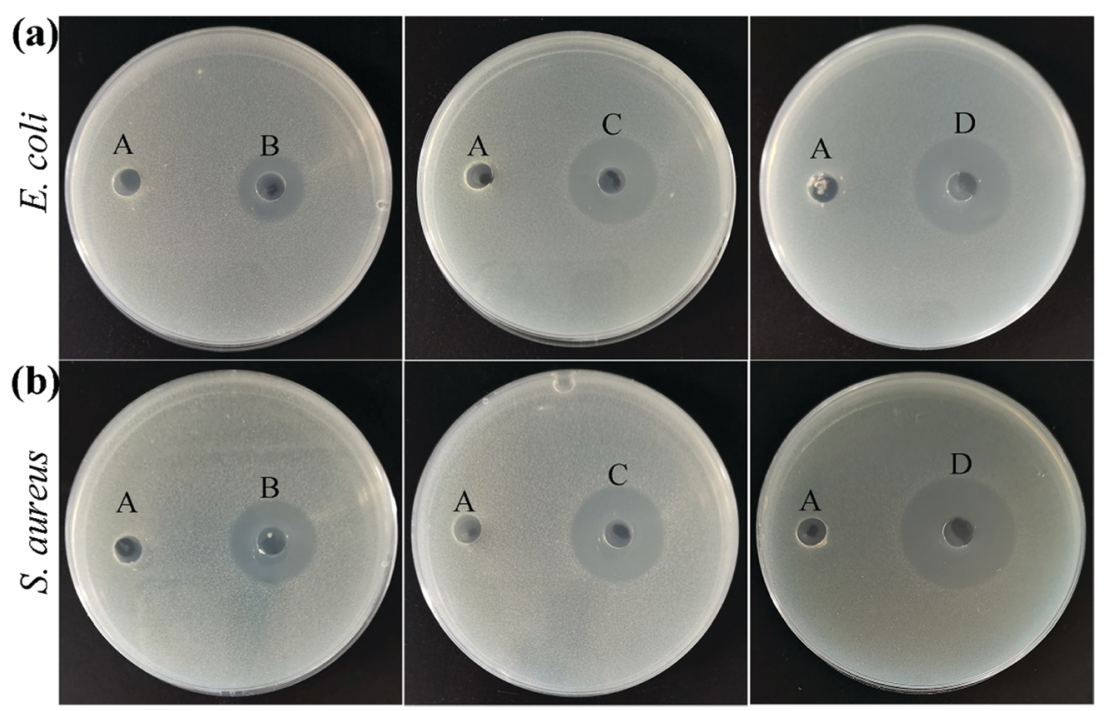

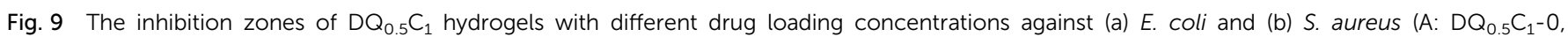
B: $\left.D_{0.5} C_{1}-10, C: D_{0.5} C_{1}-20, D: Q_{0.5} C_{1}-50\right)$. 

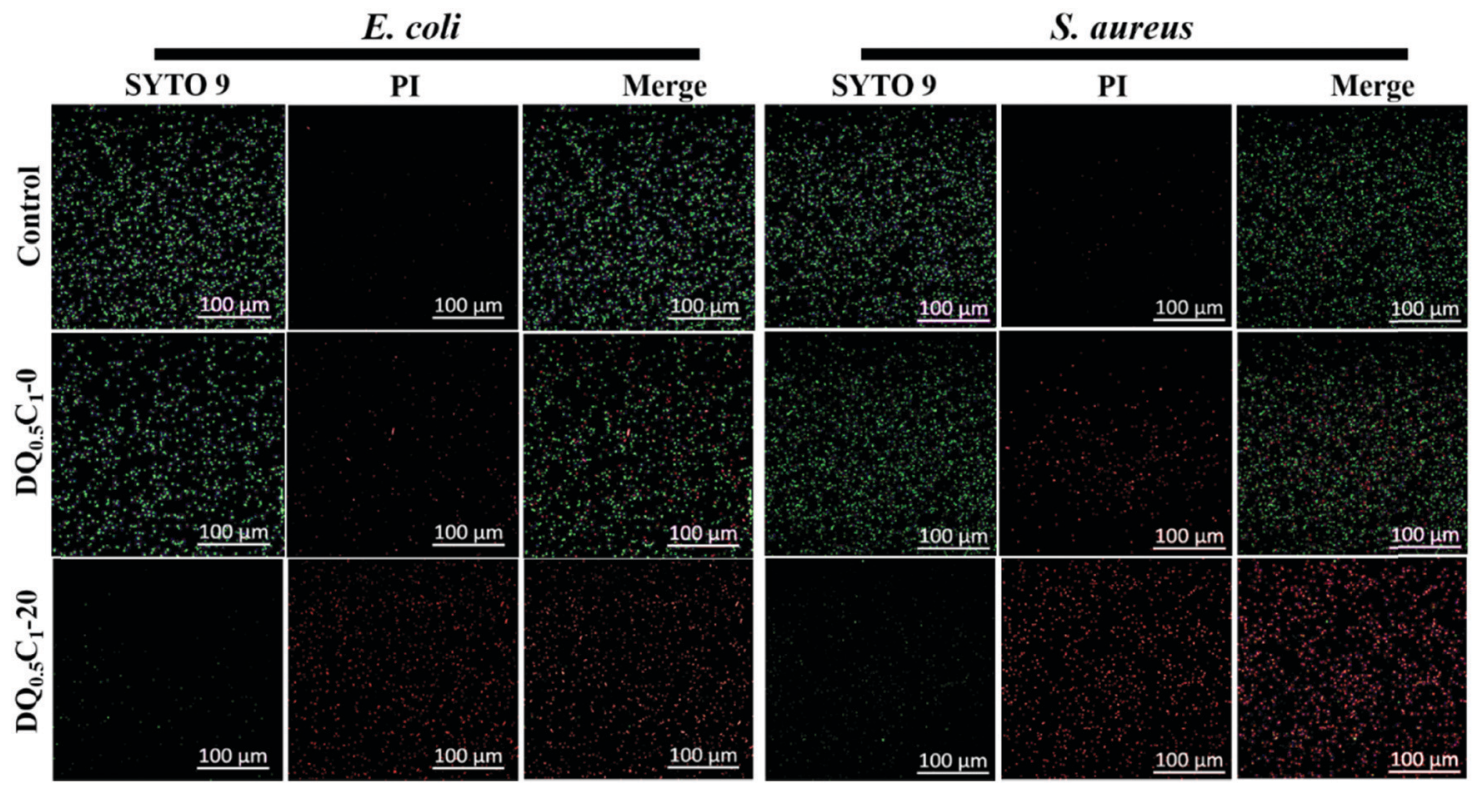

Fig. 10 Confocal fluorescent images of bacteria treated with the hydrogel carrier $\left(D Q_{0.5} C_{1}-0\right)$ and the drug-loaded hydrogel $\left(D Q_{0.5} C_{1}-20\right)$, and the assay without any treatment is used as the control.

\subsection{Inhibition zone}

In this experiment, the antibacterial properties of the hydrogels were estimated by selecting $E$. coli (Gram-negative bacteria) and S. aureus (Gram-positive bacteria) as typical bacteria. The picture and the diameter of the inhibition zone with blank control hydrogels $\left(\mathrm{DQ}_{0.5} \mathrm{C}_{1}-0\right)$ and hydrogels loaded with different drug concentrations $\left(\mathrm{DQ}_{0.5} \mathrm{C}_{1}-10, \mathrm{DQ}_{0.5} \mathrm{C}_{1}-20\right.$, and $\mathrm{DQ}_{0.5} \mathrm{C}_{1^{-}}$ 50) on E. coli and $S$. aureus are described in Fig. 9 and Table 2. After culturing at $37{ }^{\circ} \mathrm{C}$ for $24 \mathrm{~h}$, all hydrogels shrink and there is no obvious inhibition zone around the blank hydrogels while $\mathrm{DQ}_{0.5} \mathrm{C}_{1}-10, \mathrm{DQ}_{0.5} \mathrm{C}_{1}-20$, and $\mathrm{DQ}_{0.5} \mathrm{C}_{1}-50$ hydrogels are more obviously. It also can be seen from Table 2 that the diameters of the inhibition zone of $\mathrm{DQ}_{0.5} \mathrm{C}_{1}-10, \mathrm{DQ}_{0.5} \mathrm{C}_{1}-20$, and $\mathrm{DQ}_{0.5} \mathrm{C}_{1}$ 50 drug-loaded hydrogels against $E$. coli are $24.47 \pm 0.55$, $26.17 \pm 0.29$, and $29.67 \pm 0.76 \mathrm{~mm}$ and against $S$. aureus are $16.33 \pm 0.58,21.67 \pm 0.39$, and $25.00 \pm 0.50 \mathrm{~mm}$, respectively, suggesting that the prepared drug-loaded hydrogels have excellent antibacterial ability.

\subsection{Bacteria live/dead stain}

In order to observe the bactericidal effect of the hydrogels more intuitively, the fluorescent nucleic acid dyes SYTO 9 and PI were used to carry out the bacterial live/dead staining experiment. ${ }^{49}$ STYO 9 can label both live and dead bacteria, whereas PI only can reduce the intensity of SYTO 9 staining after passing through the damaged cell membrane. ${ }^{50}$ The live/dead stained image after co-cultivation with $E$. coli and $S$. aureus are illustrated in Fig. 10. The $\mathrm{DQ}_{0.5} \mathrm{C}_{1}-0$ hydrogels, after cocultivation with bacteria, show a part of red except most of green compared with the control group, indicating that the hydrogel carriers without the drug enable a certain degree of antibacterial ability, which is mainly attributed to the antibacterial ingredient QCGM. Specially, almost all of the bacteria are killed after treating with $\mathrm{DQ}_{0.5} \mathrm{C}_{1}-20$ hydrogels, demonstrating that the hydrogels, after drug loading, exhibited high antibacterial ability, which matched well with the results of the inhibition zone.

\subsection{Hemocompatibility and cell compatibility}

For biomedical material applications, hydrogels should possess good biocompatibility. The biocompatibility of $\mathrm{DQ}_{0.5} \mathrm{C}_{1}$ hydrogel was evaluated by in vitro hemolysis ${ }^{51}$ and CCK- 8 test. Firstly, the blood compatibility of the hydrogel carrier $\left(\mathrm{DQ}_{0.5} \mathrm{C}_{1}-0\right)$ and the drugloaded hydrogel $\left(\mathrm{DQ}_{0.5} \mathrm{C}_{1}-20\right)$ was tested, and the macroscopic color of centrifugally obtained supernatants and the results of hemolysis ratios for all the hydrogels group, the negative control group, and the positive control group are clearly shown in Fig. 11a. The two hydrogel groups present light yellow similar to a negative control group, while the positive group is bright red. The hemolysis rates of the hydrogels before and after drug-loading are $2.385 \%$ and $2.305 \%$ (both $<5 \%$ ), respectively, indicating that the prepared hydrogels have excellent blood compatibility. Afterwards, the influences of $\mathrm{DQ}_{0.5} \mathrm{C}_{1}$ hydrogel extract concentrations of $5,10,15,20$, and even $30 \mathrm{mg} \mathrm{mL} \mathrm{m}^{-1}$ on the activity of HSF cells were tested. Almost no decline in cell viability is observed in Fig. 11b, proving that the hydrogels exhibited excellent cell compatibility. The same conclusion can also be proved by the results shown in Fig. 11c. In general, the hemolysis and the cytotoxicity test certified that the prepared hydrogel carriers have excellent biocompatibility, which makes it show great potential in the application of wound dressing.

\subsection{In vivo wound healing}

The wound healing performance of the hydrogels was investigated by in vivo experiment using a full-thickness skin defect model. The wound images of rat at predetermined time intervals $(0,3,5,7,10$, and 14 days) are illustrated in Fig. 12a. The 
(a)
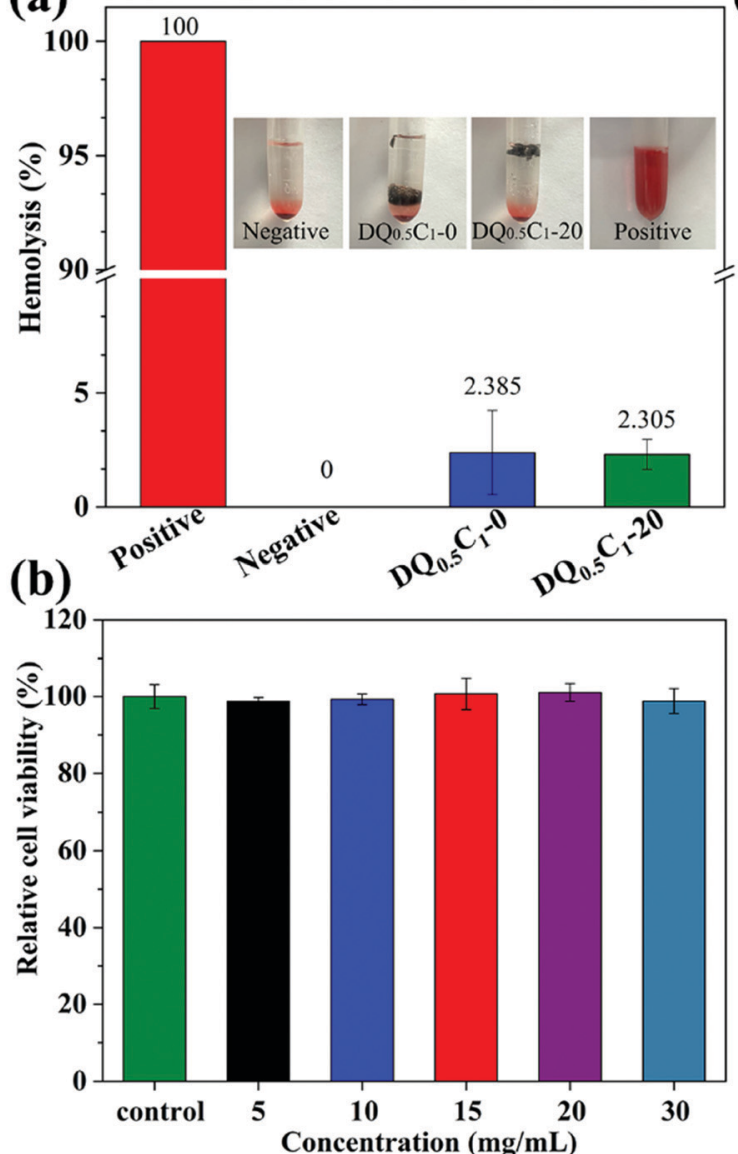

(c)
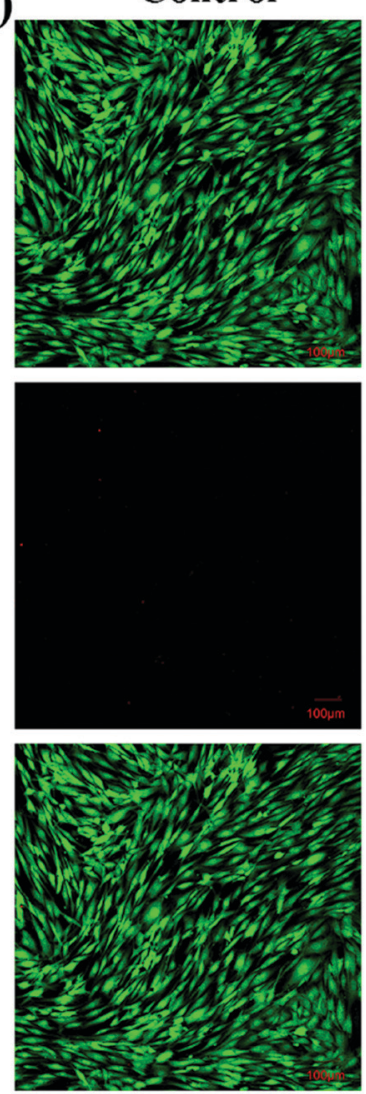

$30 \mathrm{mg} / \mathrm{mL}$
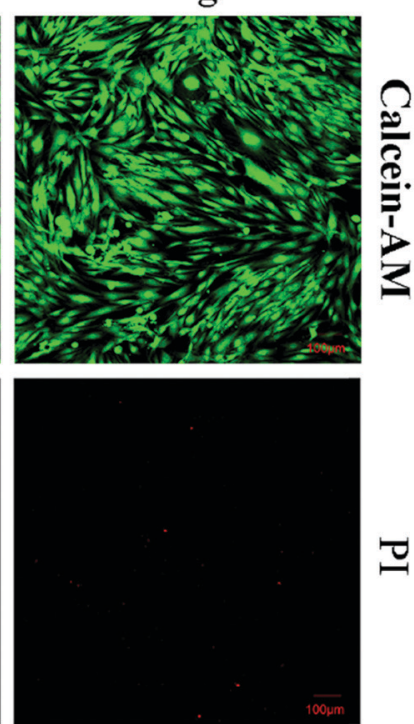

ひ

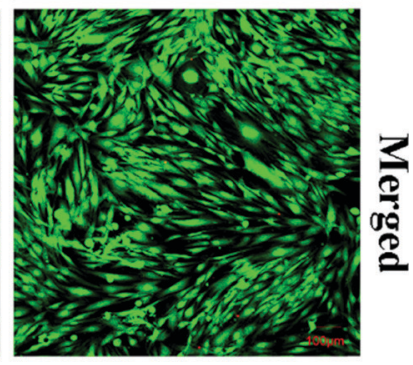

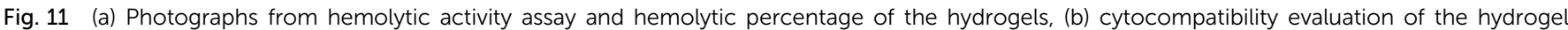

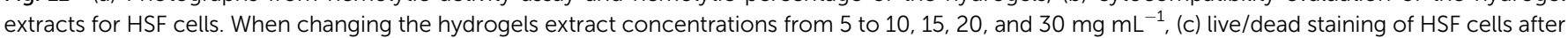
contact with the concentration of $30 \mathrm{mg} \mathrm{mL}^{-1}$ hydrogel extracts for $24 \mathrm{~h}$. Scale bar: $100 \mu \mathrm{m}$.

wound area in the hydrogel dressing group is significantly smaller than that of the blank group from three days to the end, and they were almost healed on the 10th day. The same results are also observed in Fig. 12b, which describes the degree of trauma of the wound defined as the relative value of the wound area after treatment and the original wound area at different times. The trauma of the two groups of rats both shows downward trends with the increase of treatment days, but the decline in the hydrogel groups was more obvious. HE staining is a common method to assess the quality of newly formed skin tissues. As shown in Fig. 12c, the wounds in both the control and hydrogel dressing groups healed well after 14 days of treatment, with intact skin structure and neatly arranged cells in the basal, spiny, granular, hyaline, and stratum corneum layers. The results suggest that the prepared hydrogels show enormous potential in practical applications, especially for wound healing.

\section{Conclusions}

In summary, we have successfully developed a multifunctional nanocomposite hydrogel by implanting QCGM and CNT into the DEA hydrogel systems and demonstrate that the addition of QCGM and CNT not only promoted the swelling performance and the response speed to temperature changes, but also greatly improved the mechanical properties of the hydrogels. Moreover, CNT as a photothermal agent also endows the hydrogels with excellent photothermal conversion ability so that the release of the drug is controllable by temperature or NIR light irradiation, achieving the purpose of antibacterial and anti-infection ability. Biocompatibility experiments and wound healing experiments also further manifest that the prepared-hydrogels have excellent biocompatibility and promote wound healing, which is expected to have practical applications in the field of drug delivery and wound dressings.

\section{Author contributions}

Qing Zhong: conceptualization, investigation, writing - original draft. Gen Li: investigation. Hui Long: investigation. Jingxian Zhang: methodology, formal analysis, supervision, writing - original draft, writing - review \& editing. Suiping Deng: writing - review \& editing. Langhuan Huang: conceptualization, methodology, formal analysis, 


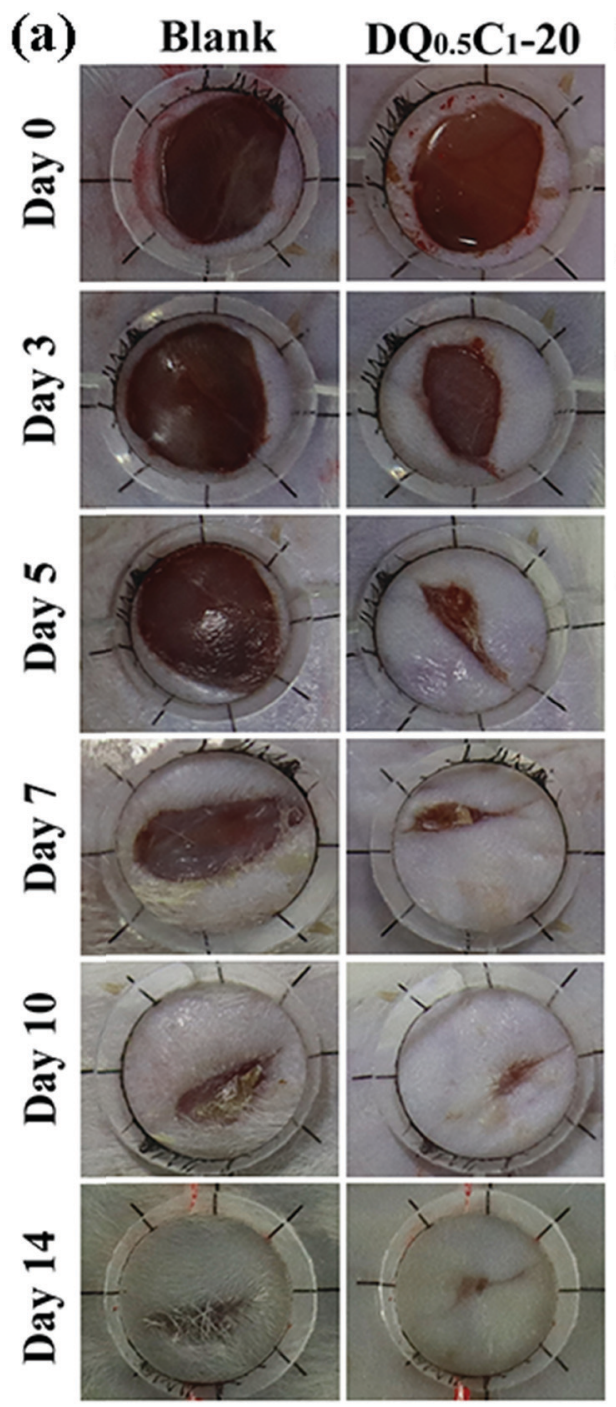

(b)

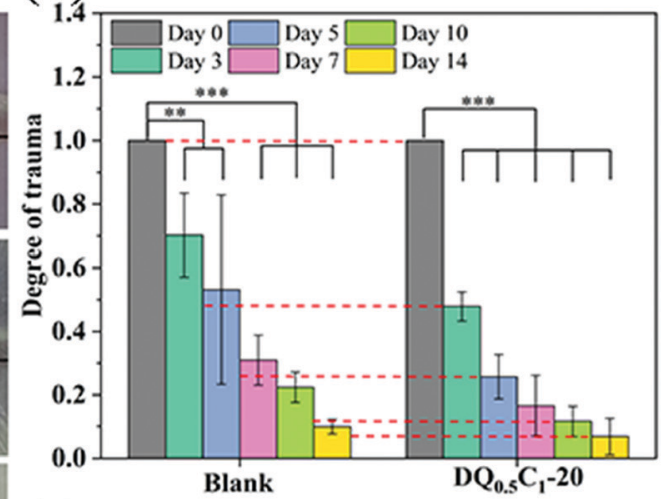

(c)
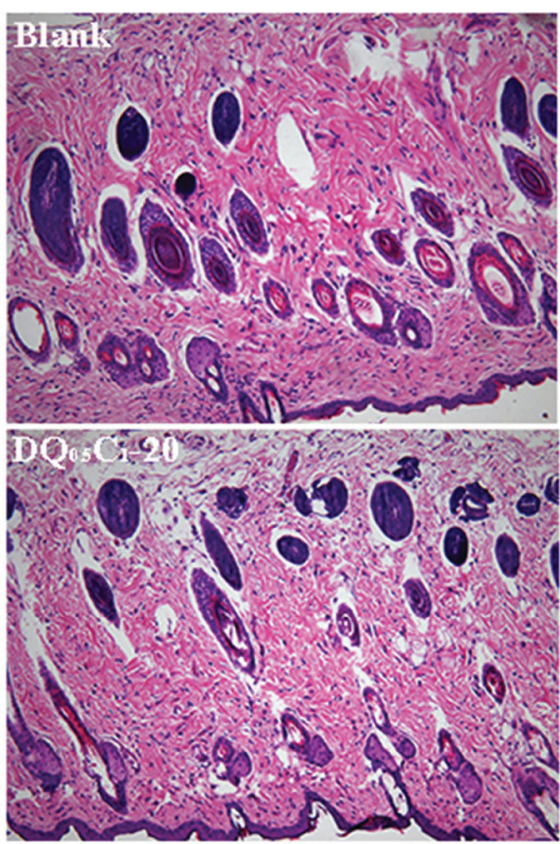

Fig. 12 (a) Photographs of wounds at 0th, 3rd, 5th, 7th, 10th, and 14th day; (b) the degree of trauma of wound; (c) histomorphological evaluation of wound sections after 14 days of treatment.

writing - original draft, writing - review \& editing. Shaozao Tan: conceptualization, writing - review \& editing, supervision, funding acquisition.

\section{Conflicts of interest}

The authors report no conflicts of interest in this work.

\section{Acknowledgements}

The authors acknowledge financial support from the National Natural Science Foundation of China (51872124), the Natural Science Key Foundation of Guangdong Province (2019B1515120056), the Innovation Team Project of Foshan City (FS0AA-KJ919-4402-0086), and the Open Experimental Project by GETRC of Graphene-like Materials and Products (GETRCGL-202005).

\section{References}

1 S. Blacklow, J. Li, B. Freedman, M. Zeidi, C. Chen and D. Mooney, Sci. Adv., 2019, 5, eaaw3963.

2 Y. Yuan, S. Shen and D. Fan, Biomaterials, 2021, 120838.

3 Y. Liang, J. He and B. Guo, ACS Nano, 2021, 15, 12687-12722.

4 Y. Wang, L. Shi, H. Wu, Q. Li, W. Hu, Z. Zhang, L. Huang, J. Zhang, D. Chen and S. Deng, ACS Omega, 2019, 4, 15373-15381.

5 B. Li, D. Li, Y. Yang, L. Zhang, K. Xu and J. Wang, Text. Res. J., 2019, 89, 801-813.

6 Y. Liang, Z. Li, Y. Huang, R. Yu and B. Guo, ACS Nano, 2021, 15, 7078-7093.

7 F. Hao, L. Wang, B. Chen, L. Qiu, J. Nie and G. Ma, ACS Appl. Mater. Interfaces, 2021, 13, 46938-46950.

8 S. Ge, J. Li, J. Geng, S. Liu, H. Xu and Z. Gu, Mater. Horiz., 2021, 8, 1189-1198. 
9 Y. Yu, Y. Cheng, J. Tong, L. Zhang, Y. Wei and M. Tian, J. Mater. Chem. B, 2021, 9, 2979-2992.

10 G. Gao, Z. Wang, D. Xu, L. Wang, T. Xu, H. Zhang, J. Chen and J. Fu, ACS Appl. Mater. Interfaces, 2018, 10, 41724-41731.

11 L. Tang, L. Wang, X. Yang, Y. Feng, Y. Li and W. Feng, Prog. Mater. Sci., 2021, 115, 100702.

12 L. Hanyková, J. Spěváček, M. Radecki, A. Zhigunov, H. Kouřilová and Z. Sedláková, Eur. Polym. J., 2016, 85, 1-13.

13 N. Işıklan and Ş. Tokmak, Carbohydr. Polym., 2019, 218, 112-125.

14 W. Wei, X. Qi, J. Li, G. Zuo, W. Sheng, J. Zhang and W. Dong, ACS Biomater. Sci. Eng., 2016, 2, 1386-1394.

15 B. Liu, J. Wang, G. Ru, C. Liu and J. Feng, Macromolecules, 2015, 48, 1126-1133.

16 M. Panayiotou, C. Pöhner, C. Vandevyver, C. Wandrey, F. Hilbrig and R. Freitag, React. Funct. Polym., 2007, 67, 807-819.

17 H. N. A. Tuan and V. T. T. Nhu, Polymers, 2020, 12, 1139.

18 Y. Liu, K. Xu, Q. Chang, M. A. Darabi, B. Lin, W. Zhong and M. Xing, Adv. Mater., 2016, 28, 7758-7767.

19 L. L. Palmese, R. K. Thapa, M. O. Sullivan and K. L. Kiick, Curr. Opin. Chem. Eng., 2019, 24, 143-157.

20 Y. Si, L. Wang, X. Wang, N. Tang, J. Yu and B. Ding, Adv. Mater., 2017, 29, 1700339.

21 T. Chen, K. Hou, Q. Ren, G. Chen, P. Wei and M. Zhu, Macromol. Rapid Commun., 2018, 39, 1800337.

22 R. Tutar, A. Motealleh, A. Khademhosseini and N. S. Kehr, Adv. Funct. Mater., 2019, 29, 1904344.

23 F. Rizzo and N. S. Kehr, Adv. Healthcare Mater., 2021, 10, 2001341.

24 Z. Deng, R. Yu and B. Guo, Mater. Chem. Front., 2021, 5, 2092-2123.

25 Z. Deng, Y. Guo, X. Zhao, P. X. Ma and B. Guo, Chem. Mater., 2018, 30, 1729-1742.

26 S. R. Shin, S. M. Jung, M. Zalabany, K. Kim, P. Zorlutuna, S. B. Kim, M. Nikkhah, M. Khabiry, M. Azize and J. Kong, ACS Nano, 2013, 7, 2369-2380.

27 X. Zhao, B. Guo, H. Wu, Y. Liang and P. X. Ma, Nat. Commun., 2018, 9, 1-17.

28 S.-Y. Ong, J. Wu, S. M. Moochhala, M.-H. Tan and J. Lu, Biomaterials, 2008, 29, 4323-4332.

29 B. Guo, R. Dong, Y. Liang and M. Li, Nat. Rev. Chem., 2021, 1-19.

30 Q. Zhong, H. Long, W. Hu, L. Shi, F. Zan, M. Xiao, S. Tan, Y. Ke, G. Wu and H. Chen, ACS Omega, 2020, 5, 8523-8533.
31 Y. Yan, C. Li, H. Wu, J. Du, J. Feng, J. Zhang, L. Huang, S. Tan and Q. Shi, ACS Appl. Bio Mater., 2019, 2, 1842-1849. 32 X. Zhao, P. Li, B. Guo and P. X. Ma, Acta Biomater., 2015, 26, 236-248.

33 P. Dhiman and M. Bhatia, Polym. Bull., 2021, 1-19.

34 S. Rashki, K. Asgarpour, H. Tarrahimofrad, M. Hashemipour, M. S. Ebrahimi, H. Fathizadeh, A. Khorshidi, H. Khan, Z. Marzhoseyni and M. SalavatiNiasari, Carbohydr. Polym., 2021, 251, 117108.

35 J. Hoque, R. G. Prakash, K. Paramanandham, B. R. Shome and J. Haldar, Mol. Pharmaceutics, 2017, 14, 1218-1230.

36 M. Li, Y. Liang, J. He, H. Zhang and B. Guo, Chem. Mater., 2020, 32, 9937-9953.

37 Y. Chen, J. Li, Q. Li, Y. Shen, Z. Ge, W. Zhang and S. Chen, Carbohydr. Polym., 2016, 143, 246-253.

38 S. Havanur, V. Farheenand and P. JagadeeshBabu, Iran. Polym. J., 2019, 28, 99-112.

39 S. Wen, T. Wu, H. Long, L. Ke, S. Deng, L. Huang, J. Zhang and S. Tan, ACS Appl. Mater. Interfaces, 2021, 13, 21979-21993.

40 G. Ovejero, J. Sotelo, M. Romero, A. Rodríguez, M. Ocana, G. Rodríguez and J. Garcia, Ind. Eng. Chem. Res., 2006, 45, 2206-2212.

41 E. Fernandez-Megia, R. Novoa-Carballal, E. Quiñoá and R. Riguera, Carbohydr. Polym., 2005, 61, 155-161.

42 J. Cho, J. Grant, M. Piquette-Miller and C. Allen, Biomacromolecules, 2006, 7, 2845-2855.

43 J. Chen, M. Liu, H. Liu and L. Ma, Mater. Sci. Eng., C, 2009, 29, 2116-2123.

44 W.-Q. Kong, C.-D. Gao, S.-F. Hu, J.-L. Ren, L.-H. Zhao and R.-C. Sun, Materials, 2017, 10, 304.

45 M. Tang, H. Dou and K. Sun, Polymer, 2006, 47, 728-734.

46 A.-J. Xie, H.-S. Yin, H.-M. Liu, C.-Y. Zhu and Y.-J. Yang, Carbohydr. Polym., 2018, 185, 96-104.

47 X. Qi, W. Wei, J. Li, Y. Liu, X. Hu, J. Zhang, L. Bi and W. Dong, ACS Biomater. Sci. Eng., 2015, 1, 1287-1299.

48 Y. Yue, H. Luo, J. Han, Y. Chen and J. Jiang, Appl. Surf. Sci., 2020, 528, 146961.

49 D. Park, J. Kim, Y. M. Lee, J. Park and W. J. Kim, Adv. Healthcare Mater., 2016, 5, 2019-2024.

50 M. T. Butler, Q. Wang and R. M. Harshey, Proc. Natl. Acad. Sci. U. S. A., 2010, 107, 3776-3781.

51 A. Sasidharan, L. S. Panchakarla, A. R. Sadanandan, A. Ashokan, P. Chandran, C. M. Girish, D. Menon, S. V. Nair, C. Rao and M. Koyakutty, Small, 2012, 8, 1251-1263. 